\title{
Neuroprotective Effect of Astersaponin I against Parkinson's Disease through Autophagy Induction
}

\author{
Lijun Zhang ${ }^{1,2,3}$, Jeoung Yun Park ${ }^{1}$, Dong Zhao ${ }^{1,2}$, Hak Cheol Kwon ${ }^{4}$ and Hyun Ok Yang ${ }^{1,2,5, *}$ \\ ${ }^{1}$ Natural Product Research Center, Korea Institute of Science and Technology, Gangneung 25451, \\ ${ }^{2}$ Division of Bio-Medical Science and Technology, KIST School, Korea University of Science and Technology, Seoul 02792, Republic \\ of Korea \\ ${ }^{3}$ State Key Laboratory for Chemistry and Molecular Engineering of Medical Resources, Guangxi Normal University, Guilin 541004, China \\ ${ }^{4}$ Natural Product Informatics Research Center, Korea Institute of Science and Technology, Gangneung 25451, \\ ${ }^{5}$ Department of Integrative Biological Sciences and Industry, Sejong University, Seoul 05006, Republic of Korea
}

\begin{abstract}
An active compound, triterpene saponin, astersaponin I (AKNS-2) was isolated from Aster koraiensis Nakai (AKNS) and the autophagy activation and neuroprotective effect was investigated on in vitro and in vivo Parkinson's disease (PD) models. The autophagy-regulating effect of AKNS-2 was monitored by analyzing the expression of autophagy-related protein markers in SHSY5Y cells using Western blot and fluorescent protein quenching assays. The neuroprotection of AKNS-2 was tested by using a 1-methyl-4-phenyl-2,3-dihydropyridium ion (MPP ${ }^{+}$)-induced in vitro PD model in SH-SY5Y cells and an MPTP-induced in vivo PD model in mice. The compound-treated SH-SY5Y cells not only showed enhanced microtubule-associated protein 1A/1B-light chain 3-II (LC3-II) and decreased sequestosome 1 (p62) expression but also showed increased phosphorylated extracellular signal-regulated kinases ( $p$-Erk), phosphorylated AMP-activated protein kinase ( $p$-AMPK) and phosphorylated unc-51-like kinase $(p-U L K)$ and decreased phosphorylated mammalian target of rapamycin ( $p$-mTOR) expression. AKNS-2-activated autophagy could be inhibited by the Erk inhibitor U0126 and by AMPK siRNA. In the MPP+-induced in vitro PD model, AKNS-2 reversed the reduced cell viability and tyrosine hydroxylase $(\mathrm{TH})$ levels and reduced the induced $\alpha$-synuclein level. In an MPTP-induced in vivo PD model, AKNS-2 improved mice behavioral performance, and it restored dopamine synthesis and TH and $\alpha$-synuclein expression in mouse brain tissues. Consistently, AKNS-2 also modulated the expressions of autophagy related markers in mouse brain tissue. Thus, AKNS-2 upregulates autophagy by activating the Erk/mTOR and AMPK/mTOR pathways. AKNS-2 exerts its neuroprotective effect through autophagy activation and may serve as a potential candidate for PD therapy.
\end{abstract}

Key Words: Autophagy, Parkinson's disease, Neuroprotection, Tyrosine hydroxylase, Motor symptoms

\section{INTRODUCTION}

Parkinson's disease (PD) is the second most common neurodegenerative disease after Alzheimer's disease. The central pathological feature of PD is the loss of dopaminergic neurons in the substantia nigra pars compacta (SNpc). Lewy bodies (LBs) form due to the abnormal accumulation of $\alpha$-synuclein in the neurons is another neuropathology of PD (Poewe et al., 2017). Moreover, abnormal motor symptoms are frequently found in PD patients (Dauer and Przedborski, 2003).

1-Methyl-4-phenyl-1,2,3,6-tetrahydropyridine (MPTP), is a potent neurotoxin that can destroy dopaminergic neurons in the SNpc (Kostrzewa, 2014). In many kinds of animals, MPTP produces severe and irreversible symptoms similar to those in PD. Once MPTP crosses the blood-brain barrier (BBB), it can be converted to the real toxin 1-methyl-4-phenylpyridinium $\left(\mathrm{MPP}^{+}\right.$) by monoamine oxidase type $\mathrm{B}$ (MAO-B). A portion of $\mathrm{MPP}^{+}$enters mitochondria and blocks the mitochondrial electron transport enzyme NADH dehydrogenase, thus inhibits cellular respiration (Ramsay and Singer, 1986; Klaidman et al., 1993). Exposure to MPTP also leads to the pathological aggregation of proteins, including ubiquitin and $\alpha$-synuclein, and the aggregated proteins cannot be metabolized (Conway et al., 2000). MPTP is applied and considered the best experi-

\section{Open Access https://doi.org/10.4062/biomolther.2021.004}

This is an Open Access article distributed under the terms of the Creative Commons Attribution Non-Commercial License (http://creativecommons.org/licenses/by-nc/4.0/) which permits unrestricted non-commercial use, distribution, and reproduction in any medium, provided the original work is properly cited.
Received Jan 6, 2021 Revised May 17, 2021 Accepted Jun 7, 2021

Published Online Jul 2, 2021

\section{*Corresponding Author}

E-mail: hoyang@sejong.ac.kr

Tel: +82-2-3408-1959, Fax: +82-2-3408-4336 
mental tool to mimic the specific features of PD.

Autophagy is a self-degradative process, and it is important to balance energy sources and adapt to nutrient stress. Autophagy is a crucial survival mechanism and plays a key role in treating various diseases, including neurodegeneration diseases (Glick et al., 2010), it can be regulated through a complicated signaling pathway. mTOR complex 1 (mTORC1) reflects cellular nutritional status and links nutrient signals to autophagy regulation (Kim et al., 2011). The inhibition of mTORC1 by rapamycin induces autophagy in the presence of nutrients, which demonstrates that TOR is an autophagy inhibitor (Noda and Ohsumi, 1998). AMP-activated protein kinase (AMPK) plays a role in cellular energy homeostasis. AMPK can be activated by low energy levels through the regulation of the upstream kinase LKB1, thereby activating the TSC1/2 complex. The activation of TSC1/2 leads to the inhibition of mTOR activity through inactivating the TOR activator Rheb (He and Klionsky, 2009). AMPK can directly activate ULK1 by upregulating phosphorylation, while it also regulates ULK1 activity through mediating the activity of TSC2 and mTOR1 (Inoki et al., 2003; Egan et al., 2011). Autophagy can also be up-regulated under nutrient starvation through Erk1/2 activation, Erk1/2 activates autophagy through mTORC1 inhibition via binding to and activating TSC, the depletion of Erk can partially inhibit autophagy (Pattingre et al., 2003; Wang et al., 2009). Numerous studies indicate that the age-related decline in autophagy disturbs neuronal homeostasis, thus leading to the deposition of toxic components in the cytoplasm and subsequently the progression of neurodegenerative disorders such as PD. Postmortem examinations of brains from PD patients show the distinct accumulation of autophagosomes and loss of lysosomal markers in dopaminergic neurons (Chu et al., 2009; Dehay et al., 2010).

Aster koraiensis Nakai (AKNS), is commonly distributed in the southern and central parts of the Korean peninsula and Jeju Island (Prabakaran et al., 2017). AKNS is used in traditional Korean medicine for treating diseases such as diabetes, chronic bronchitis, pertussis, and pneumonia (Hyun et al., 2018; Kim et al., 2018). Recently, research by our team reported that a compound, triterpene saponin, astersaponin I (AKNS-2) was isolated from AKNS (Kwon et al., 2019), the present research tried to study the effect AKNS-2 on autophagy induction and elucidate the underlying pathways, eventually explore its protective effect on PD.

\section{MATERIALS AND METHODS}

\section{Isolation of active compound AKNS-2}

The process of Aster koraiensis extraction and compound isolation is described in another published research (Kwon et al., 2019). Briefly the plant was ground by a pulverizer, and then was extracted with $95 \% \mathrm{EtOH}$ solution. Next, the powdered product (named AKNS extract) was extracted by using $n$-hexane, EtOAc and $n-\mathrm{BuOH}$, respectively. Subsequently, $n-\mathrm{BuOH}$ fraction was isolated using a reversed phase HPLC (YMC-Triart C18 column). Finally the mixture of target compounds was separated by Sephadex ${ }^{\circledR}$ LH-20 (GE Healthcare, Chicago, IL, USA). One of the active compounds was purified and the structure was identified as $3-O-\beta-D-$ xylopyranosyl-( $1 \rightarrow 3)-\beta$-D-glucopyranosylpolygalacic acid 28 O- $\alpha$-L-rhamnopyranosyl-( $1 \rightarrow 3)-\beta-D-x y l o p y r a n o s y l-(1 \rightarrow 4)$ $[\beta$-D-xylopyranosyl-( $1 \rightarrow 3)]$ - $\alpha$-L-rhamnopyranosyl- $(1 \rightarrow 2)$ $\alpha$-L-arabinopyranosyl ester, which termed astersaponin I (AKNS-2), using NMR (500 MHz) (showed as Fig. 1).

\section{Materials}

Dulbecco's modified Eagle's medium (DMEM), fetal bovine serum (FBS), 100 units $/ \mathrm{mL}$ penicillin and $100 \mathrm{mg} / \mathrm{mL}$ streptomycin are purchased from Gibco (Thermo Fisher ScientificWaltham, MA, USA). 1-Methyl-4-pheynl-1,2,3,6-tetrahydropyridine (MPTP) hydrochloride, 1-Methyl-4-phenylpyridinium $\left(\mathrm{MPP}^{+}\right)$iodide, 3-methyladenine (3-MA) and ropinirole were purchased from Sigma Chemical Co (St. Louis, MO, USA). Wortmannin (Wort) and bafilomycin A1 (Baf) are from Abcam (MA, USA). Anti-glyceraldehyde-3-phosphate dehydrogenase (GAPDH), anti-AMPK, anti-phosphorylated AMPK (p-AMPK), anti-ULK, anti-phosphorylated ULK555 (p-ULK555), anti-Erk, anti-phosphorylated Erk (p-Erk), anti-mTOR, anti- phosphorylated mTOR (p-mTOR), anti-LC3B, anti-tyrosine hydroxylase (TH), anti- $\alpha$-synuclein primary antibodies sourced from rabbit, anti-rabbit horseradish peroxidase-conjugated IgG secondary antibody, the Erk inhibitor U0126, the AMPK siRNA, AMPK siRNA control were purchased from Cell Signaling Technology (Boston, MA, USA). An autophagy Tandem Sensor RFP-GFPLC3B kit was from Thermo Fisher Scientific. MTT assay kit ZCytox was from DAEILLAB Co, Ltd (Seoul, Korea). Dopamine ELISA kit was from Abnova (Taipei City, Taiwan) and the MAOB assay kit was supplied by Promega (Woods Hollow Road Madison, WI, USA). All other chemicals were of the highest

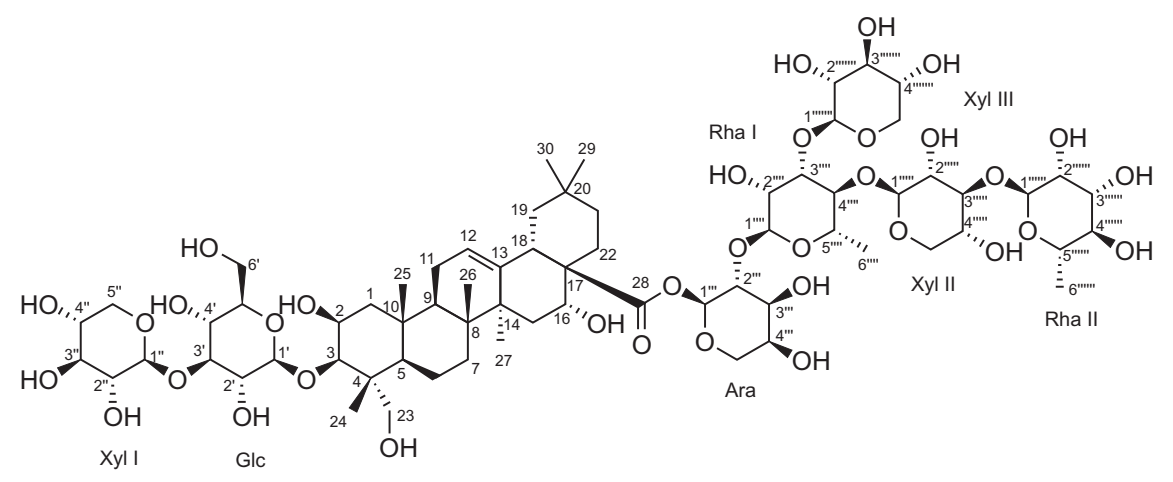

AKNS-2

Fig. 1. Chemical structure of the active compound AKNS-2. 
grade and were found from commercial resources.

\section{Cell culture}

Human neuroblastoma cells of SH-SY5Y were supplied by the company of American Type Culture Collection (Manassas, VA, USA), and were cultured in DMEM supplemented with $10 \%$ heat-inactivated FBS and $1 \%$ penicillin/streptomycin. $\mathrm{SH}-\mathrm{SY} 5 \mathrm{Y}$ cells were incubated under condition of $37^{\circ} \mathrm{C}$ in humidified atmosphere with $5 \% \mathrm{CO}_{2}$. First cells were seeded on 6 -well plate at a density of $80 \times 10^{4}$ cells/well in $2 \mathrm{~mL}$ medium, after $24 \mathrm{~h}$ were treated with samples AKNS-2 at the desired concentrations. Under the conditions of 3-MA, Wort, Baf or U0126 presence, cells were treated with these reagents 30 min prior to sample treatment. For AMPK siRNA transfected SH-SY5Y cells, AKNS-2 should be administered $36 \mathrm{~h}$ after siRNA transfection. When $\mathrm{MPP}^{+}$treatment was needed, cells should be treated with MPP ${ }^{+} 1 \mathrm{~h}$ after sample treatment. Twenty-four $\mathrm{h}$ after sample treatment, SH-SY5Y cells were harvested and used for further analysis.

\section{MTT assay for measurement of cell viability}

Cell viability was detected by using a MTT assay kit Z-Cytox (DAEILLAM Co, Ltd, Seoul, Korea). Briefly, SH-SY5Y cells were seeded on a 96-well plate at a density of $2 \times 10^{4}$ cells/ well in $100 \mu \mathrm{L}$ medium. Twenty-four $\mathrm{h}$ later, to determinate the cytotoxicity of AKNS-2 and $\mathrm{MPP}^{+}, \mathrm{SH}-\mathrm{SY} 5 \mathrm{Y}$ cells were treated with AKNS-2 and/or MPP+ at desired concentrations. After $24 \mathrm{~h}, 10 \mu \mathrm{L}$ of MTT reagent was added to each cell containing well of 96 -well plate. The absorbance at $450 \mathrm{~nm}$ was measured using a microplate spectrophotometer (BioTek, VT, USA). In order to verify the protective effect of AKNS-2 against $\mathrm{MPP}^{+}$induced cytotoxicity, we treated cells with $2 \mathrm{mM} \mathrm{MPP}^{+} 1$ $\mathrm{h}$ after the AKNS-2 treatment. The next day, the absorbance was measured $1 \mathrm{~h}$ after addition of MTT reagent. To elucidate if AKNS-2 exerts its protective effect on $\mathrm{MPP}^{+}$-induced cytotoxicity through autophagy induction, we treated $\mathrm{SH}-\mathrm{SY} 5 \mathrm{Y}$ cells with U0126 or AMPK siRNA, followed by AKNS-2 (5 and $10 \mu \mathrm{M})$ addition $30 \mathrm{~min}$ or $36 \mathrm{~h}$ later, respectively, $1 \mathrm{~h}$ after AKNS treatments, cells were added $2 \mathrm{mM} \mathrm{MPP}^{+}$.

\section{Measurement of RFP-GFP-LC3 by fluorescence microscope}

SH-SY5Y cells were cultured on glass coverslips in 24-well plates at a density of $8 \times 10^{4} /$ well. The transfection process were conducted according to the instruction of the Autophagy Tandem Sensors RFP-GFP-LC3B kit (Invitrogen, Carlsbad, CA, USA). After cultivation for $24 \mathrm{~h}$, cells were treated with LC3B reagent. The transfected cells were incubated for $24 \mathrm{~h}$, then $50 \mathrm{nM}$ Wort or $100 \mathrm{nM}$ Baf was administered followed by the addition of AKNS-2 $(10 \mu \mathrm{M})$. The cells were further fixed with $4 \%$ paraformaldehyde and then permeabilized with $0.1 \%$ Triton X100. Nuclei were stained with 4,6-diamidino-2-phenylindole (DAPI, $25 \mu \mathrm{g} / \mathrm{mL}$ ). Fluorescence signals were detected using a confocal microscope (Leica, Solms, Germany).

\section{Preparation of cell lysates}

Twenty-four $h$ after drug treatment, medium in the wells of 6 -well plate was discarded and the cells were gently rinsed one time using cold saline. Next, $1 \mathrm{~mL}$ cold saline was added to each well, cells adhered to the bottom were suspended by scouring the bottom with a $1 \mathrm{~mL}$ pippet. The suspension liquids were collected in $1.5 \mathrm{~mL}$ tubes and centrifuged at $13000 \mathrm{xg}$, $4^{\circ} \mathrm{C}$ for $5 \mathrm{~min}$. After supernatants removal, cell pellets were added $50 \mu \mathrm{L}$ of RIPA lysis buffer from Cell Signaling Technology containing protease inhibitor cocktail (Roche, Mannheim, Germany). After shaking at $4^{\circ} \mathrm{C}$ for 30 min, obtained cell samples were centrifuged at $13000 \mathrm{xg}, 4^{\circ} \mathrm{C}$ for $20 \mathrm{~min}$. The supernatants were collected and the protein concentrations were measured by Bradford method with a BSA constructed standard curve. Subsequently, supernatants were diluted with loading buffer, and then heated at $99^{\circ} \mathrm{C}$ for $5 \mathrm{~min}$. The resultant cells samples were used for further Western blot analysis.

\section{Animals}

All the animal care and experimental protocols in the present study complied with the guidelines of the Korea Institute of Science and Technology Animal Care Committee (no. 2017018). All efforts were made to minimize the mice number and relieve their suffering. Forty mice (C57BL/6j, male, 8 weeks old) were ordered from Japan SLC Inc (Shizuoka, Japan). After arrival, four mice were fed in each cage $(30 \times 18.5 \times 13$ $\mathrm{cm}$ ) and were supplied with unlimited food and water. All mice were housed under the following constant conditions: lights on from $6: 00$ to $18: 00$, temperature of $23 \pm 1{ }^{\circ} \mathrm{C}$, and humidity of $50 \pm 10 \%$. After a habituation period of 7 days, the mice were subjected to a series of experimental operations according to standard protocols.

\section{Animal grouping and sample treatment}

Forty mice were divided into 5 groups randomly, with 8 mice in each group. After the habituation period, all mice were orally administered a single dose of drug each day. The mice in group 1 and group 2 were administered saline (p.o.), while the mice in group 3, group 4, and group 5 were administered $5 \mathrm{mg} / \mathrm{kg}$ ropinirole (p.o.), $5 \mathrm{mg} / \mathrm{kg}$ AKNS-2 (p.o.), and $15 \mathrm{mg} / \mathrm{kg}$ AKNS2 (p.o.), respectively. Here dopamine agonist ropinirole was applied as a positive control. From day 5 , the mice in group 1 were intraperitoneally injected with saline $1 \mathrm{~h}$ after saline gavage, and the mice in groups 2, 3, 4 and 5 were administered $30 \mathrm{mg} / \mathrm{kg}$ MPTP (i.p.) $1 \mathrm{~h}$ after the saline, ropinirole, AKNS-2 $5 \mathrm{mg} / \mathrm{kg}$ and AKNS-2 $15 \mathrm{mg} / \mathrm{kg}$ gavage, respectively. Each mouse received a single dose of saline/MPTP injection for 8 days. Seven days after the last MPTP injection, all mice were killed by cervical dislocation, and the whole brain, SNpc and striatum (ST) were dissected for further biochemical analysis.

\section{Rotarod test}

The rotarod test was conducted following the description (Hu et al., 2017a) with minor modifications. Briefly, the test consisted of a pretraining section and a test section. The pretraining section was performed over 4 consecutive days. All mice were placed on the cylinder of the rotarod apparatus with the tail towards the operator, and then the training was started at a constant speed of $16 \mathrm{rpm}$ over a period of $300 \mathrm{~s}$. During this $300 \mathrm{~s}$, the mice that fell to the ground were placed back on the cylinder by the operator. All mice were subjected to a total of 3 trials within a 30-min interval each day before MPTP administration. Beginning on the day after the last behavioral training day, we treated mice with a single dose of $30 \mathrm{mg} / \mathrm{kg}$ MPTP for 8 consecutive days $1 \mathrm{~h}$ after AKNS-2 (5 and $15 \mathrm{mg} /$ $\mathrm{kg}$ ) administration. The latency of falling to the ground was recorded for each mouse. For the test section, the performance of all mice on the rotarod was tested following the protocol used during the pretraining section at the 3 time points of 2 $\mathrm{h}, 24 \mathrm{~h}$ and $48 \mathrm{~h}$ after the last injection of MPTP. The latency 
of each mouse to fall to the ground was recorded. Only mice that remained on the cylinder longer than $60 \mathrm{~s}$ in the pretraining section were used in the statistical analysis. The average time of the three trials was calculated to evaluate balance, grip strength and motor coordination.

\section{Pole test}

A pole test was conducted following the description in a study (Choi et al., 2013) with minor modifications. Briefly, on the day before MPTP administration, a wooden pole $(50 \mathrm{~cm}$ in length, $1 \mathrm{~cm}$ in diameter) with a rough surface was set up in a sound-proof room. Mice were placed on the top of the pole with its head arranged skyward, and the time mice took to turn around and climb down the pole was recorded, up to a maximum of $120 \mathrm{~s}$, by a stopwatch. The same training operation was performed in triplicate within an interval of $30 \mathrm{~min}$. After MPTP injection, the performance of all mice on the pole was tested using the same protocol as in pretraining at the 3 time points of $2 \mathrm{~h}, 24 \mathrm{~h}$ and $48 \mathrm{~h}$ after the last MPTP injection. The average time of three trials was analyzed to assess motor function.

\section{Wire hanging test}

The wire hanging test was performed according to the study (Zhu et al., 2018) with modification. Briefly, a horizontal wire $(1.5 \mathrm{~mm}$ in diameter, $50 \mathrm{~cm}$ in length and $30 \mathrm{~cm}$ above the bedding material) was fixed between two poles. Soft bedding was placed underneath the wire. A mouse was handled by the tail and allowed to grasp the middle point of the wire with its forepaws. The timer was started immediately after the proper suspension of the mouse. The time until the mouse fell down from the wire was recorded, up to a maximal duration of $300 \mathrm{~s}$. On the day before MPTP injection, each mouse was subjected to 3 trials, and the average hanging time of 3 trials was analyzed as an index to evaluate balance, muscle function and coordination. At $2 \mathrm{~h}, 24 \mathrm{~h}$, and $48 \mathrm{~h}$ after MPTP injection, we tested mouse performance on the wire hanging task. The average latency of each mouse to fall down to the bedding material was calculated.

\section{Preparation of mouse brain tissue}

Seven days after the last injection of MPTP, the SNpc and ST of mice were carefully dissected and stored at $-80^{\circ} \mathrm{C}$ before use. Subsequently, the brain tissues of the SNpc and ST were homogenized in PRO-PREPTM lysis buffer (iNtRON, Gyeonggi, Korea) containing Phosphatase Inhibitor Cocktail Set I (Sigma-Aldrich, MO, USA). After shaking at $4^{\circ} \mathrm{C}$ for 30 $\mathrm{min}$, the homogenates were centrifuged at $13000 \times \mathrm{g}$ and $4^{\circ} \mathrm{C}$ for $20 \mathrm{~min}$, and then the obtained supernatants were collected in a new $1.5 \mathrm{~mL}$ tube. The protein concentrations in the supernatants were measured using the Bradford method. A portion of supernatant was mixed with an equal volume of loading buffer and denatured on a $99^{\circ} \mathrm{C}$ heater for $5 \mathrm{~min}$ for Western blot analysis. The remaining supernatants were stored at $-80^{\circ} \mathrm{C}$ for further analysis of MAO-B activity and DA level by ELISA kits (MAO-B activity ELISA kits, Promega, Madison, WI, USA; DA level ELISA kits, Abnova).

\section{Measurement of DA level}

DA levels in the ST were measured by a competitive ELISA kit (Abnova) following the manufacturer's instructions. Briefly, the $\mathrm{ST}$ was homogenized in $0.01 \mathrm{~N} \mathrm{HCl}$ in the presence of
EDTA and sodium metabisulfite. The homogenate was centrifuged at $13000 x g$ for $5 \mathrm{~min}$. The supernatants were collected and used to measure DA level. After the determination of the protein concentrations in the supernatants, the DA levels in each brain sample were detected in duplicate using an ELISA kit (Abnova). The absorbance at $450 \mathrm{~nm}$ was detected by a microreader (BioTek), and the intensity was inversely proportional to the DA level. The DA level is expressed as $\mathrm{ng} / \mathrm{mg}$ protein.

\section{Determination of MAO-B activity}

The MAO-B activity of the ST and SN was determined by a MAO-B assay kit from Promega according to the manufacturer's instructions. The kit applied a homogeneous luminescent method for detecting MAO activity. The assay includes 2 steps. First, the MAO-B substrate was added to MAO-B enzyme-containing material (ST and SN samples) to generate methyl ester luciferin; second, the produced methyl ester luciferin reacted with esterase and luciferase to produce light. The MAO-B activity was directly proportional to the amount of developed light. The luminescent signal was measured using an Infinite M1000 multimode microplate reader (TECAN, Männedorf, Switzerland). MAO-B activity was expressed as relative light units $(\mathrm{RLU}) / \mathrm{mg}$ protein.

\section{Western blot analysis}

The protein markers in the lysates from SH-SHY5Y cells and mouse brain tissues (SNpc and ST) were measured using Western blot analysis. Briefly, after determining the concentration, protein samples $(20 \mu \mathrm{g})$ were separated by $8 \%, 10 \%$ or $15 \%$ sodium dodecyl sulfate-polyacrylamide gel electrophoresis (SDS-PAGE). Then, the separated proteins on the gel were transferred onto a polyvinylidene fluoride (PVDF) buffered saline with $0.1 \%$ Tween 20 (TBST) for $5 \mathrm{~min}$, the membrane was blocked with $5 \%$ skim milk dissolved in TBST buffer. The membrane was further incubated with monoclonal primary antibodies sourced from rabbits (including anti-GAPDH, anti-AMPK, anti-p-AMPK, anti-ULK, anti-p-ULK555, anti-Erk, anti-p-Erk, anti-mTOR, anti-p-mTOR, anti-LC3B, anti-p62, anti-TH and anti- $\alpha$-synuclein antibodies). Primary antibodies were diluted to a ratio of $1: 1000$ in blocking buffer and incubated at $4{ }^{\circ} \mathrm{C}$ overnight. On the second day, membrane was washed with TBST 3 times (10 min each time) and then incubated in horseradish peroxidase-conjugated goat anti-rabbit IgG secondary antibodies (diluted to a ratio of 1:2000 in blocking buffer) at room temperature. After incubation in secondary antibody for $1 \mathrm{~h}$, the membrane was washed for $30 \mathrm{~min}$. The protein blots on the membrane were developed with an ECL detection kit and visualized using a LAS-4000 mini system (Fujifilm, Tokyo, Japan). Once the protein blot signal saturated, LAS-4000 mini system automatically paints it red color and we stop the detection. In order to accurately quantify the intensity of target protein, only the band showing unsaturated signal should be used for the quantitative analysis (Ghosh et al., 2014). The present research chose the unsaturated bands and quantified the intensities of protein with Multi Gauge V3.0 software (Fujifilm).

\section{Statistical analysis}

All the data in this study were analyzed using GraphPad Prism 7 software (GraphPad Software, CA, USA) and presented as mean \pm SEM. Statistical analysis were carried out using one-way analysis of variance (ANOVA) or Student's $t$-test. A 
value of $p<0.05$ was considered statistical significance.

\section{RESULTS}

\section{AKNS-2 activated autophagy through the mTOR-dependent signaling pathway}

To investigate the autophagy-inducing effects of AKNS-2, SH-SY5Y cells were treated with AKNS-2 at 5, 10, or $20 \mu \mathrm{M}$. After treatment for $24 \mathrm{~h}$, the expression levels of autophagy-related protein markers were measured by Western blot analysis. As shown in Fig. 2A, AKNS-2 treatment induced the expression of LC3-II, a key autophagy indicator, in SH-SY5Y cells in a dose-dependent manner. Moreover, AKNS-2 treatment at 10 and $20 \mu \mathrm{M}$ significantly increased p-Erk expression, while treatment with 5,10 , and $20 \mu \mathrm{M}$ AKNS-2 led to significant increases in p-AMPK and p-ULK expression. p-mTOR expression was inhibited by 10 and $20 \mu \mathrm{M}$ AKNS-2. These findings indicate that AKNS-2 may upregulate autophagy by activating the AMPK/mTOR pathway and/or the Erk/mTOR pathway.

3-Methyladenine (3-MA) inhibits the formation of autophagosome (Pattingre et al., 2003). To confirm the autophagy activation induced by AKNS-2, we employed 3-MA (5 mM) to disturb the autophagosome accumulation $30 \mathrm{~min}$ before treating SH-SY5Y cells with $10 \mu \mathrm{M}$ AKNS-2. The results suggested $10 \mu \mathrm{M}$ AKNS-2 significantly increased LC3-II expression (as shown in Fig. 2B), however this enhancement was significantly inhibited by 3-MA; p62 also plays critical role in autophagy, it binds to LC3 through a region called the LC3-interacting region (LIR) and can be degraded when autophagy is activated
(Bjørkøy et al., 2009; Moscat and Diaz-Meco, 2009). Interestingly, AKNS-2 also induced a significant reduction in $\mathrm{p} 62$ expression (Fig. 2B), and 3-MA treatment markedly blocked the inhibitory effect of AKNS-2 on p62 level. In a tandem fluorescent protein quenching assay, accumulation of GFP-RFPLC3-II puncta (Fig. 2C) was evaluated using an autophagy sensor from Thermo Fisher Scientific. More cytoplastic puncta stained by green fluorescence and red fluorescence were observed in the transfected cells treated with only AKNS-2 than in the control cells. To confirm that AKNS-2 enhanced autophagy, transfected cells were cotreated with AKNS-2 and the autophagy inhibitors wortmannin (Wort; $50 \mathrm{nM}$ ) or bafilomycin $\mathrm{A}_{1}$ (Baf; $100 \mathrm{nM}$ ). As a the PI3K inhibitor, wortmannin inhibits autophagy through blocking the formation of autophagosome (Wu et al., 2010), while bafilomycin $A_{1}$ prevents lysosomal acidification, thus interfering with the degradation of autophagosome (Yang et al., 2013; Yoshii and Mizushima, 2017). AKNS-2-enhanced accumulation of green and red LC3-II puncta was reduced by Wort, while Baf increased green fluorescence and decreased red fluorescence. These findings demonstrated that AKNS-2 activated autophagy.

\section{AKNS-2 protects SH-SY5Y cells against MPP+-induced neurotoxicity by activating autophagy}

We tried to investigate the protective effects of AKNS-2 on an $\mathrm{MPP}^{+}$-induced in vitro PD model. Fig. 3A shows that AKNS2 did not significantly affect cytotoxicity up to the high concentration of $40 \mu \mathrm{M}$ by MTT assay. The working concentrations of $\mathrm{MPP}^{+}$and AKNS-2 were also determined by an MTT assay. Fig. 3B shows that $\mathrm{SH}-\mathrm{SY} 5 \mathrm{Y}$ cells were treated with $\mathrm{MPP}^{+}$at various concentrations, and $\mathrm{MPP}^{+}$at $2 \mathrm{mM}$ significantly re-
A
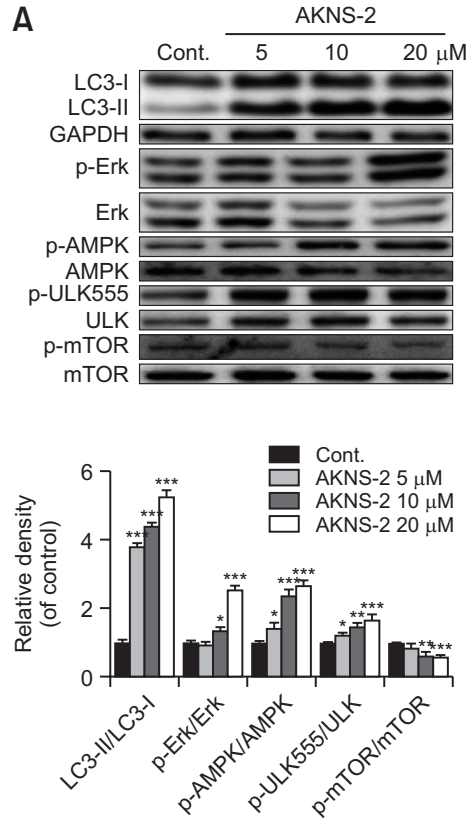

B
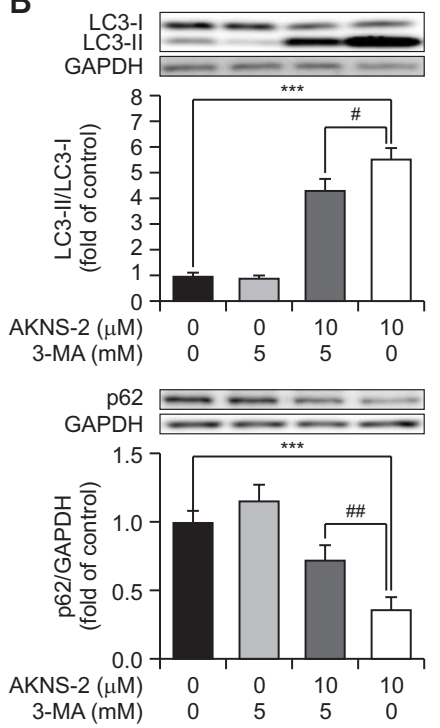

C

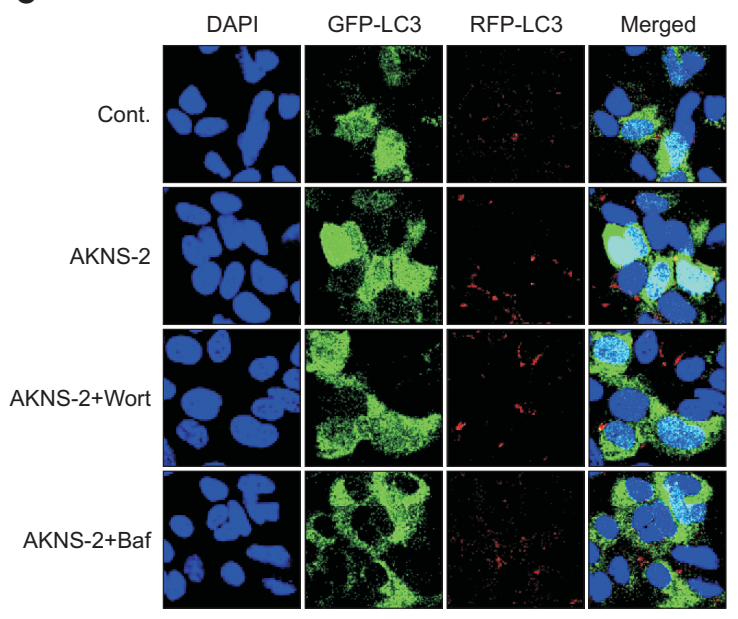

Fig. 2. AKNS-2 activated autophagy through mTOR dependent signals pathway. Twenty-four $h$ after treatment with AKNS-2, key markers involved in autophagy induction in SH-SY5Y cells were measured by Western blot method. (A) shows the expressions of autophagy related protein markers in SH-SY5Y cells treated with AKNS-2. (B) shows AKNS-2 up-regulated LC3-II and reduced p62 expressions were reversed by 3-MA (5 mM). (C) mRFP-GFP-LC3 Tandem Fluorescent Protein Quenching Assay indicates AKNS-2 induced autophagy was inhibited by wortmannin (Wort, $50 \mathrm{nM}$ ) and bafilomycin $\mathrm{A}_{1}$ (Baf, $100 \mathrm{nM}$ ). The data are expressed as mean $\pm \operatorname{SEM}(n=3)$. ${ }^{*} p<0.05,{ }^{* *} p<0.01,{ }^{* * *} p<0.001$ significant difference from control, ${ }^{\#} p<0.05,{ }^{\#} p<0.01$ significant difference from AKNS-2 treated group. 
A

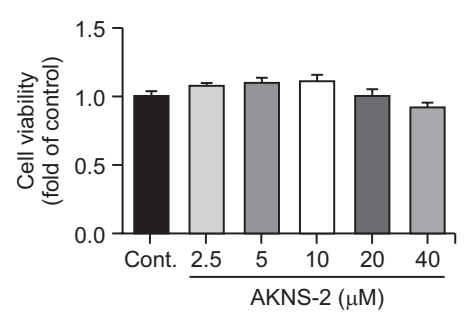

D

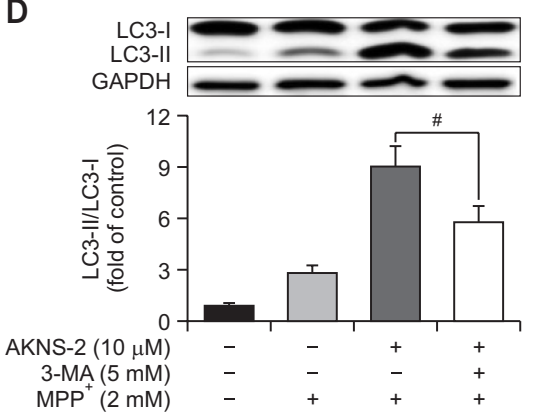

B

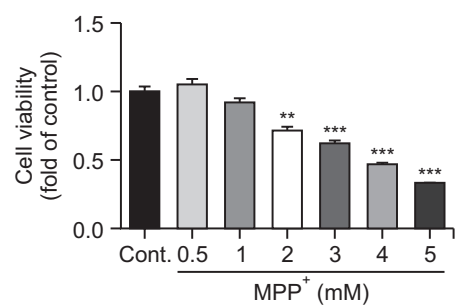

$E$

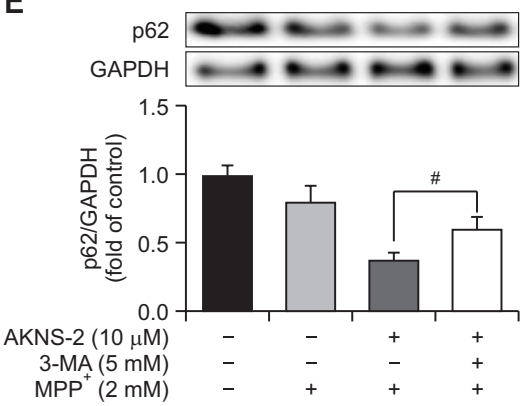

C

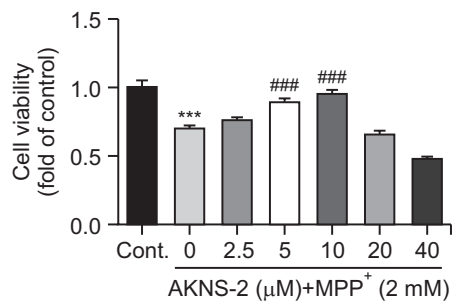

$\mathrm{F}$

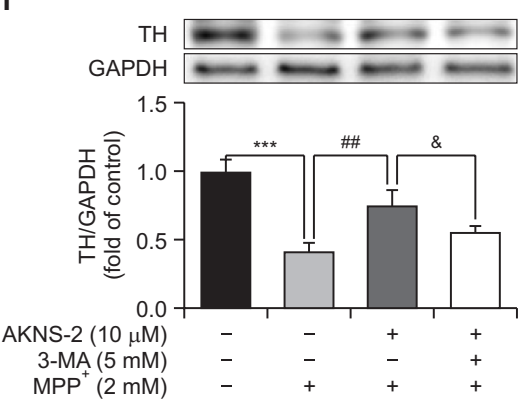

Fig. 3. Protective effect of AKNS-2 on SH-SY5Y cells from MPP+-induced toxicity was inhibited by autophagy inhibitor 3-MA. (A), (B), (C) show the cell viabilities of SH-SY5Y cells after treatment with AKNS-2 only, MPP ${ }^{+}$only, AKNS-2 and MPP together, respectively, at various concentrations. (D), (E), (F) show the protein expressions of LC3, p62, TH respectively, in SH-SY5Y cells treated with AKNS-2 and MPP in the absence or presence of 3-MA. The data are expressed as mean \pm SEM ( $n=5$ for MTT assay, and $n=3$ for western blot analysis). ${ }^{* *} p<0.01,{ }^{* * *} p<0.001$ significant difference from control, ${ }^{\#} p<0.05,{ }^{\# \#} p<0.01,{ }^{\# \#} p<0.001$ significant difference from MPP ${ }^{+}$treated group. ${ }^{\&} p<0.05$ significant difference from AKNS-2 and MPP ${ }^{+}$group.

duced cell viability. Therefore, we decided to induce an in vitro PD model using $2 \mathrm{mM} \mathrm{MPP}^{+}$. Next, we tested the protective effect of AKNS-2 against MPP ${ }^{+}$induced neurotoxicity. Briefly, $\mathrm{SH}-\mathrm{SH} 5 \mathrm{Y}$ cells were treated with AKNS-2 at various concentrations, and after $1 \mathrm{~h}, 2 \mathrm{mM} \mathrm{MPP}{ }^{+}$was added to the cells. Cell viability was detected $24 \mathrm{~h}$ after treatment. The results (Fig. $3 \mathrm{C}$ ) showed that 5 and $10 \mu \mathrm{M}$ AKNS-2 markedly enhanced the cell viability impaired by $2 \mathrm{mM} \mathrm{MPP}$.

To investigate whether AKNS-2 exerted a protective effect on MPP+-damaged SH-SY5Y cells by activating autophagy. We employed an autophagy inhibitor, 3-MA, to block the autophagy activated by AKNS-2. Compared to the AKNS-2 and $\mathrm{MPP}^{+}$cotreated group, the 3-MA-treated group had significantly inhibited LC3-II expression (Fig. 3D), while the p62 (Fig. $3 \mathrm{E})$ decrease caused by $\mathrm{MPP}^{+}$and AKNS-2 was restored by 3-MA. Thus, the autophagy activated by AKNS-2 was blocked by 3-MA. The enzyme tyrosine hydroxylase $(\mathrm{TH})$ is expressed throughout the central nervous system. TH converts tyrosine to L-3,4-dihydroxyphenylalanine (L-DOPA), which can be processed into DA. TH is the rate-limiting enzyme of DA synthesis. Interestingly, in addition to inhibiting autophagy, the $\mathrm{MPP}^{+}-$ induced decrease in $\mathrm{TH}$ (as Fig. 3F) expression was reversed by AKNS-2 treatment, and the beneficial effect of AKNS-2 was also abolished by 3-MA treatment.

\section{AKNS-2 upregulates autophagy and protects against $\mathrm{MPP}^{+}$neurotoxicity in SH-SY5Y cells by activating the Erk/ mTOR pathway}

A study reported that the depletion of Erk can partially inhibit autophagy and that the activation of Erk inhibits mTORC1 by binding to and activating TSC, thus upregulating autophagy (Wang et al., 2009). U0126 is an inhibitor of the kinase MEK1/2; U0126 prevents the activation of Erk1/2 and can be used to investigate the role of Erk in autophagy induction. To elucidate whether AKNS-2 activates autophagy by modulating the Erk/mTOR signal pathway, SH-SY5Y cells were treated with AKNS-2 (5 and $10 \mu \mathrm{M})$ in the absence or presence of U0126 $(10 \mu \mathrm{M})$. Twenty-four $\mathrm{h}$ after cultivation, Western blot analysis was employed to evaluate the induction of autophagy and the expression of protein markers involved in the Erk-modulated autophagy pathway. The results are shown in Fig. 4. LC3-II expression was significantly increased by AKNS-2 (5 and 10 $\mu \mathrm{M})$ treatment compared with the control condition. When 10 $\mu \mathrm{M} \cup 0126$ was added 30 min before AKNS-2 treatment, a significant reduction in LC3-II expression (Fig. 4A) was found in both the 5 and $10 \mu \mathrm{M}$ AKNS-2-treated groups, which indicates that AKNS-2-induced autophagy was inhibited by U0126. A significant increased expression in p-Erk (Fig. 4B) was found in both the 5 and $10 \mu \mathrm{M}$ AKNS-2-treated groups compared with the control group, while the increased expression of p-Erk in $10 \mu \mathrm{M}$ AKNS-2-treated cells was dramatically abolished by $10 \mu \mathrm{M}$ U0126 administration. Afterwards, p-mTOR expression (Fig. 4D) was measured. Compared with the control condition, AKNS-2 (10 $\mu \mathrm{M})$ significantly inhibited p-mTOR expression, and when $10 \mu \mathrm{M}$ U0126 was administered 30 min before AKNS-2 treatment, the inhibition of p-mTOR expression induced by AKNS-2 ( 5 and $10 \mu \mathrm{M}$ ) was markedly recovered. AKNS-2 (5 and $10 \mu \mathrm{M}$ ) significantly enhanced p-ULK555 expression, as shown in Fig. 4C, while $10 \mu \mathrm{M}$ U0126 abolished the enhancing effect of $10 \mu \mathrm{M}$ AKNS-2 on p-ULK. Here, we concluded that AKNS-2 upregulates autophagy in SH-SY5Y cells by activating the Erk/mTOR pathway.

$\mathrm{TH}$ is the rate-limiting enzyme of DA synthesis. $\alpha$-synuclein is the primary constituent of the LB, which is one of the pathologic features of PD. The present research aimed to study the protective effect of AKNS-2 on $\mathrm{MPP}^{+}$-induced neurotoxicity in 
A

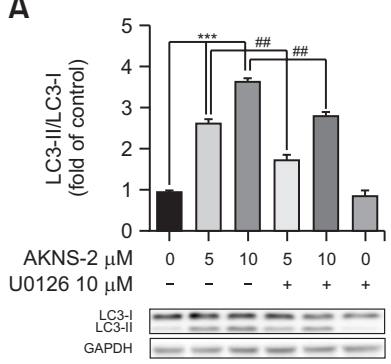

E

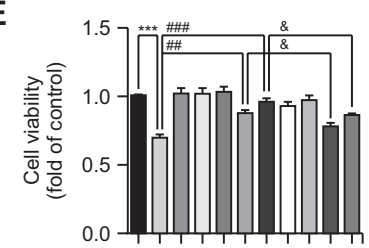

AKNS-2 $\mu \mathrm{M} \quad 0 \quad 0 \quad 0 \quad 5510510510510$ $\mathrm{MPP}^{+} 2 \mathrm{mM}-+-+++-++$ $\mathrm{U} 012610 \mu \mathrm{M}-+_{+-+++++}$
B

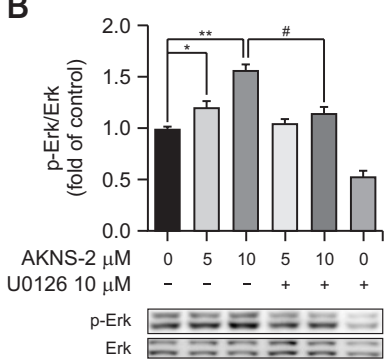

F

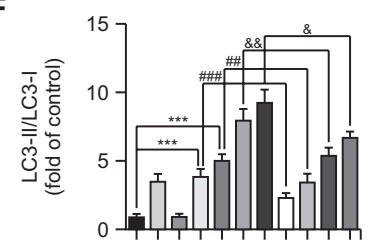

AKNS-2 $\mu$ M 0000510510510510 $\mathrm{MPP}^{+} 2 \mathrm{mM}-+-\cdot++-++$ $\mathrm{U} 012610 \mu \mathrm{M}$ LC3-1
LCCS-II
GAPDH
C

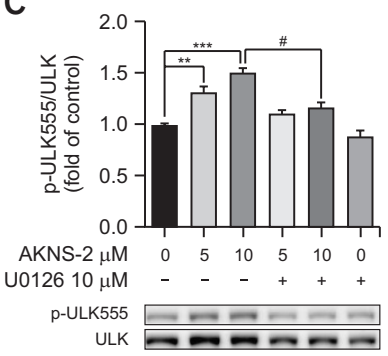

G

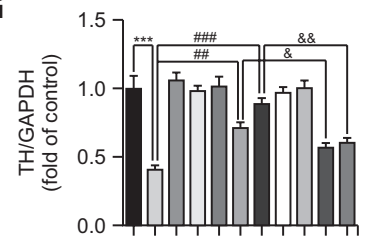

AKNS-2 $\mu$ M 0000510510510510 $\mathrm{MPP}^{+} 2 \mathrm{mM}$ U0126 $10 \mu \mathrm{M}$

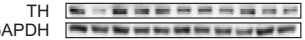

D

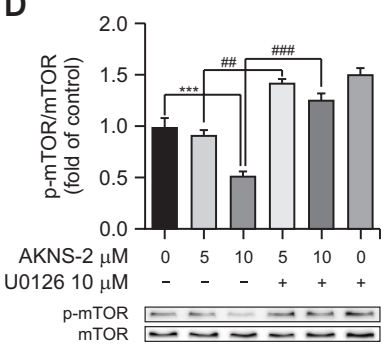

$\mathrm{H}$

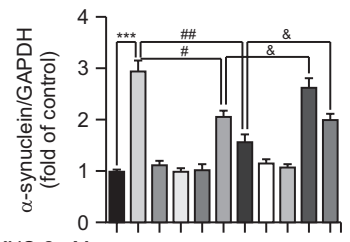

AKNS-2 $\mu \mathrm{M} \quad 0 \quad 0 \quad 0 \quad 510510510510$ $\mathrm{MPP}^{+} 2 \mathrm{mM}-+\cdots++\cdots++$ U0126 $10 \mu \mathrm{M}$

$\alpha$-synuclein

Fig. 4. AKNS-2 exerts its protective effect on SH-SY5Y cells from MPP ${ }^{+}$induced toxicity through activating Erk signal pathway. SH-SY5Y cells were treated with AKNS-2 (5 and $10 \mu \mathrm{M})$ in the presence or absence of $10 \mu \mathrm{M}$ U0126, (A), (B), (C), (D) show the protein expressions of LC3, Erk, ULK, mTOR, respectively. The data are expressed as mean \pm SEM $(n=3)$. ${ }^{*} p<0.05,{ }^{* *} p<0.01$, ${ }^{* * *} p<0.001$ significant difference from control, ${ }^{\#} p<0.05,{ }^{\#} p<0.01,{ }^{\# \#} p<0.001$ significant difference from AKNS-2 treated group. SH-SY5Y cells were treated with AKNS-2 and $\mathrm{MPP}^{+}(2 \mathrm{mM})$ in the presence or absence of $10 \mu \mathrm{M} U 0126$, (E) shows U0126 abolished the protective effect of AKNS-2 on cell viability; ( $\left.\mathrm{F}\right)$, $(\mathrm{G})$, (H) show the expressions of LC3, TH and $\alpha$-synuclein. The data are expressed as mean \pm SEM ( $n=5$ for MTT assay, and $n=3$ for western blot analysis). ${ }^{* * *} p<0.001$ significant difference from control, ${ }^{\#} p<0.05,{ }^{\#} p<0.01,{ }^{\# \#} p<0.001$ significant difference from MPP ${ }^{+}$treated group (for LC3 difference from AKNS-2 treated group). ${ }^{\circledR} p<0.05,{ }^{\& \&} p<0.01$ significant difference from AKNS-2 and MPP ${ }^{+}$treated group.

SH-SY5Y cells. Cell viability was examined by MTT assay. The results in Fig. 4E indicate that AKNS-2 at 5 and $10 \mu \mathrm{M}$ significantly enhanced the decreased cell viability induced by $2 \mathrm{mM} \mathrm{MPP}^{+}$, but this enhancement was abolished by U0126 treatment. Next, we examined the expression of LC3 (Fig. 4F). The results showed that U0126 blocked the enhancing effect of AKNS-2 (5 and $10 \mu \mathrm{M}$ ) on LC3-II expression; similarly, the increase in LC3-II expression due to AKNS-2 and $\mathrm{MPP}^{+} \mathrm{Co}-$ treatment was also abolished by U0126 administration. These results confirmed that AKNS-2 enhanced cell viability and that AKNS-2 enhanced autophagy and cell viability by modulating Erk signaling. Moreover, TH expression (Fig. 4G) was significantly reduced by $2 \mathrm{mM} \mathrm{MPP}$, while the $\mathrm{TH}$ reduction could be recovered by AKNS-2 at 5 and $10 \mu \mathrm{M}$. When U0126 was administered before AKNS-2 and $\mathrm{MPP}^{+}$treatment, the enhancing effect of AKNS-2 on TH expression was inhibited. Regarding $\alpha$-synuclein expression, $2 \mathrm{mM} \mathrm{MPP}^{+}$significantly increased $\alpha$-synuclein expression (as Fig. $4 \mathrm{H}$ ) in $\mathrm{SH}-\mathrm{SY} 5 \mathrm{Y}$ cells, and AKNS-2 treatment counteracted the impact of MPP ${ }^{+}$ and inhibited $\alpha$-synuclein expression. Interestingly, the protective effect was blocked in the presence of U0126. AKNS-2 reversed the altered expression of $\mathrm{TH}$ and $\alpha$-synuclein and enhanced the decreased cell viability by $\mathrm{MPP}^{+}$treatment in SH-SY5Y cells; however, these protective effects could be inhibited by disturbing autophagy by blocking the Erk signaling pathway. These facts suggest that AKNS-2 exerts its protective effect on $\mathrm{MPP}^{+}$induced cytotoxicity via activating autophagy by regulating Erk signal.

\section{AKNS-2 upregulates autophagy and protects against $\mathrm{MPP}^{+}$neurotoxicity in SH-SY5Y cells by activating the AMPK/mTOR pathway}

AMPK activation leads to the activation of TSC $1 / 2$, thereby inhibiting mTOR activity through inactivating the TOR activator Rheb, which eventually activates ULK1 and autophagy. (Sarkar, 2013). To investigate whether AKNS-2 upregulates autophagy by modulating the AMPK/mTOR pathway, we employed an AMPK siRNA (50 nM) to disturb AMPK signaling in SH-SY5Y cells, and then SH-SY5Y cells were treated with AKNS-2 (5 and $10 \mu \mathrm{M})$. Twenty-four $\mathrm{h}$ after AKNS-2 treatment, representative protein markers involved in the AMPK/ mTOR signaling pathway that modulates autophagy, including LC3, AMPK, mTOR and ULK, were measured by Western blot analysis. Significant increases in LC3-II (Fig. 5A), p-AMPK (Fig. 5B) and p-ULK555 (Fig. 5C) were found in the AKNS-2 treated group compared with the control group. The AKNS2-enhanced expression of LC3-II, p-AMPK and p-ULK555 was dramatically reduced in AMPK siRNA-transfected SH-SY5Y cells compared with normal AKNS-2-treated SH-SY5Y cells. Additionally, AKNS-2 significantly inhibited p-mTOR expression (Fig. 5D), but the inhibition was abolished in response to disturbance of AMPK signaling.

Next, we tried to elucidate whether AKNS-2 protects SHSY5Y cells from MPP+-induced cytotoxicity and to explore the role of AMPK-mediated autophagy in this protection. After $\mathrm{SH}$ SY5Y cells were transfected with 50 nM AMPK siRNA, the cells were treated with AKNS-2 $(5$ and $10 \mu \mathrm{M})$ in the absence or presence of $2 \mathrm{mM} \mathrm{MPP}{ }^{+}$. Cell viability was test by MTT assay, and as shown in Fig. 5E, significantly decreased cell 
A

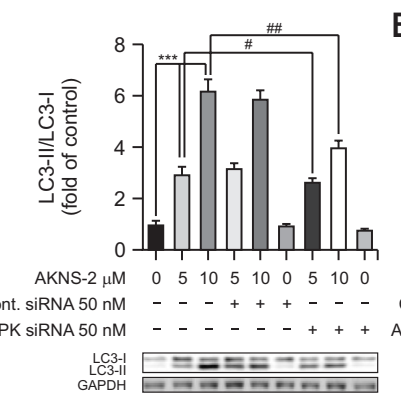

B

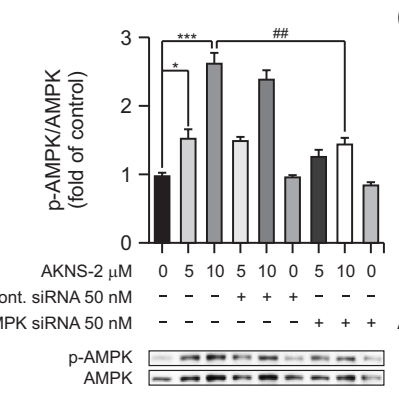

C

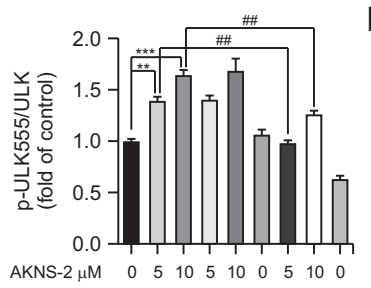

D

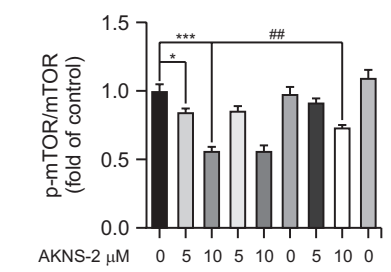
Cont. siRNA $50 \mathrm{nM} \ldots \ldots+\ldots+\ldots$ Cont. siRNA $50 \mathrm{nM}-\ldots+\ldots+\ldots$ AMPK SIRNA $50 \mathrm{nM}+\ldots$ - $-\ldots++$ AMPK SIRNA $50 \mathrm{nM}$

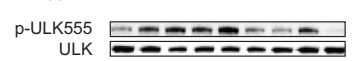
p-mTOR
mTOR

E

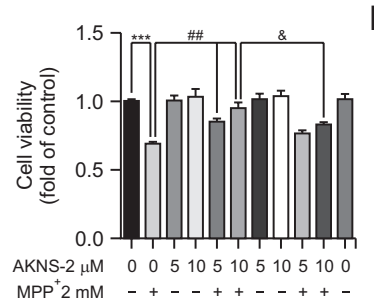

$\mathrm{MPP}^{+} 2 \mathrm{mM}-+-++-++$
F

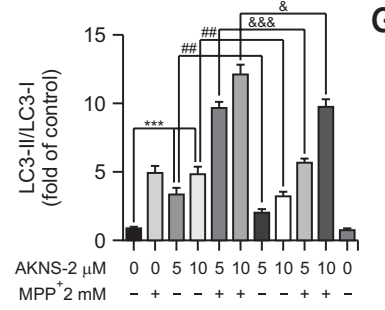

MPP $2 \mathrm{mM}-+-++-+++-$

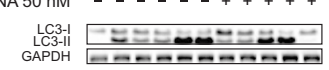

G

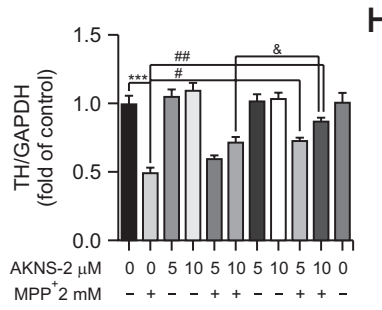

MPP $2 \mathrm{mM}-+\cdots++-++-$
AMPK SiRNA $50 \mathrm{nM}-{ }^{-++++\cdots}+\cdots+$

$\mathrm{H}$

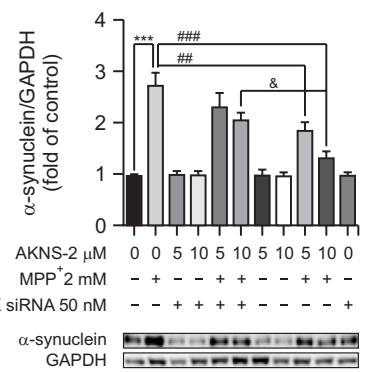

Fig. 5. AKNS-2 exerts its protective effect on SH-SY5Y cells from MPP ${ }^{+}$induced toxicity through activating AMPK signal pathway. SHSY $5 Y$ cells were treated with AKNS-2 $(5$ and $10 \mu \mathrm{M})$ in the presence or absence of AMPK siRNA (50 nM), (A), (B), (C), (D) show the protein expressions of LC3, AMPK, ULK, mTOR, respectively. The data are expressed as mean \pm SEM $(n=3) .{ }^{*} p<0.05,{ }^{* *} p<0.01,{ }^{* * *} p<0.001$ significant difference from control, ${ }^{\#} p<0.05,{ }^{\#} p<0.01$ significant difference from only AKNS-2 treated group. SH-SY $5 Y$ cells were treated with AKNS-2 and $\mathrm{MPP}^{+}(2 \mathrm{mM})$ in the presence or absence of AMPK siRNA, (E) shows AKNS-2 increased cell viability reduced by MPP ${ }^{+}, \mathrm{AMPK}$ siRNA abolished the protective effect of AKNS-2; (F), (G), (H) show the typical protein bands of LC3, TH, $\alpha$-synuclein and relative densities. The data are expressed as mean \pm SEM ( $n=5$ for MTT assay, and $n=3$ for western blot analysis). ${ }^{* * *} p<0.001$ significant difference from control, ${ }^{\#} p<0.05,{ }^{\# p<0.01, ~}{ }^{\# \#} p<0.001$ significant difference from MPP ${ }^{+}$treated group (for LC3 difference from AKNS-2 treated group). ${ }^{\&} p<0.05$, \&\&\& $p<0.001$ significant difference from AKNS-2 and MPP ${ }^{+}$treated group.

viability was observed with $\mathrm{MPP}^{+}$treatment compared with the control condition, and AKNS-2 $(5$ and $10 \mu \mathrm{M})$ reversed the effect of $\mathrm{MPP}^{+}$on cell viability. The viability of AMPK siRNAtransfected SH-SY5Y cells was significantly lower than that of normal SY-SY5Y cells in the presence of ANKS-2 and MPP+ Regarding the expression of protein markers, LC3-II (Fig. 5F) expression in normal SH-SY5Y cells was markedly enhanced by AKNS-2 ( 5 and $10 \mu \mathrm{M})$, but the LC3-II level in AMPK siRNA-transfected cells was markedly lower than that in normal SH-SY5Y cells in the presence of AKNS-2. Similarly, LC3-II expression was significantly reduced in siRNA-transfected cells after AKNS-2 and MPP ${ }^{+}$cotreatment compared with normal cells. Moreover, TH expression (Fig. 5G) was significantly reduced by $\mathrm{MPP}^{+}$administration, and AKNS-2 treatment counteracted the toxicity of $\mathrm{MPP}^{+}$and reversed $\mathrm{TH}$ expression. Interestingly, the AKNS-2-mediated improvement in $\mathrm{TH}$ expression was abolished by AMPK siRNA. MPP ${ }^{+}$significantly increased $\alpha$-synuclein expression (Fig. $5 \mathrm{H}$ ), and the increase in $\alpha$-synuclein level was inhibited by AKNS- 2 in normal cells. However, in AMPK siRNA-transfected cells, the inhibition of $\alpha$-synuclein by AKNS-2 was abolished. These facts indicate that AKNS-2 upregulated autophagy by modulating AMPK signaling and that activated autophagy protects SH-SY5Y cells from $\mathrm{MPP}^{+}$-induced toxicity.

\section{AKNS-2 improved the behavioral performance of an MPTP-induced in vivo PD model}

We also tested the protective effect of AKNS-2 on an MPTPinduced in vivo PD model (Fig. 6A showed the scheme). As a non-ergot dopamine agonist, ropinirole delays the motor disorders and is effective in treating early PD (Nashatizadeh et al., 2009). In the present study, ropinirole was employed as a positive control to evaluate the protection of AKNS-2 on the induced in vivo PD model. First, we tested the effect of AKNS2 on MPTP-impaired behavioral performance using rotarod test, pole test and wire hanging test. After training, the mice were administered MPTP once daily for 8 consecutive days. The behavioral performance in the rotarod test, pole test and wire hanging test were examined at 2, 24, and $48 \mathrm{~h}$ after the last MPTP injection. The mouse behavioral performance in the rotarod test was markedly impaired in the MPTP-treated group compared with the normal group at 2 and $24 \mathrm{~h}$ after injection, but the impairment was reversed by $5 \mathrm{mg} / \mathrm{kg}$ ropinirole and 15 $\mathrm{mg} / \mathrm{kg}$ AKNS-2 at 2 and $24 \mathrm{~h}$. Treatment with $5 \mathrm{mg} / \mathrm{kg}$ AKNS-2 significantly improved the MPTP-impaired performance in the rotarod test at $24 \mathrm{~h}$. At $48 \mathrm{~h}$, the performance of all mice was recovered to the normal level (as shown in Fig. 6B). MPTP treatment significantly damaged performance in the pole test at $2 \mathrm{~h}$ after injection; however, the damaged performance was significantly improved by ropinirole and AKNS-2 administration (5 and $15 \mathrm{mg} / \mathrm{kg}$ ) at $2 \mathrm{~h}$. No significant differences were found among all groups at 24 and $48 \mathrm{~h}$ after MPTP injection (as shown in Fig. 6C). For the wire hanging test, compared with the control condition, MPTP injection significantly decreased the latency to fall at $2 \mathrm{~h}$ and $24 \mathrm{~h}$. At $48 \mathrm{~h}$, the MPTP-induced decrease in latency was recovered to the level in pretraining. The positive control ropinirole $(5 \mathrm{mg} / \mathrm{kg}$ ) significantly improved the latency impaired by MPTP at $2 \mathrm{~h}$, while the impaired per- 
A AKNS-2 5 and 15 , and ropinirole $5 \mathrm{mg} / \mathrm{kg} / \mathrm{day}$
for 12 days

\begin{tabular}{|c|c|c|c|}
\hline$\underbrace{\begin{array}{c}\text { Behavior } \\
\text { pre-training }\end{array}}$ & $\begin{array}{c}\begin{array}{c}\text { MPTP injection } \\
30 \mathrm{mg} / \mathrm{kg} / \text { day, } 8 \text { days }\end{array} \\
.\end{array}$ & $\overbrace{}^{\begin{array}{c}\text { Behavior } \\
\text { test }\end{array}}$ & $\begin{array}{c}\text { Dissection of SNpc and ST } \\
\& \\
\text { biochemical assay }\end{array}$ \\
\hline & & $\perp$ & 1 \\
\hline
\end{tabular}
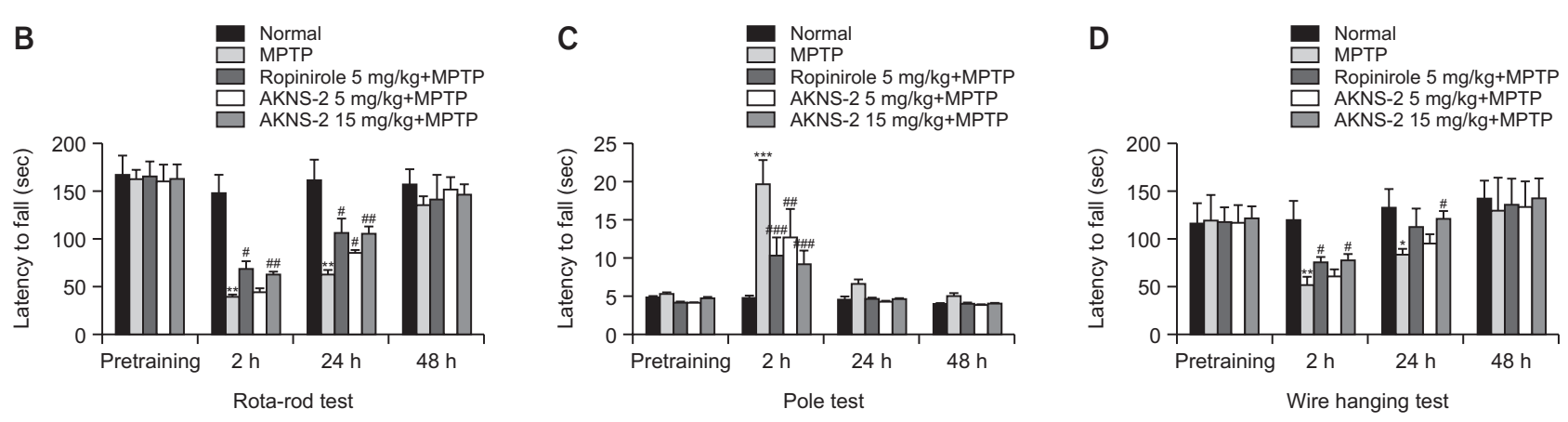

Fig. 6. Protective effect of AKNS-2 on MPTP impaired animal behavioral performance. (A) shows the scheme of sub-chronic MPTP administration-induced in vivo PD model. Mice were pre-trained in Rota-rod, Pole and Wire hanging test for 3 days. After the last MPTP injection, mice behavior performances were recorded at different time points. (B), (C) and (D) show the behavior performances of mice in Rota-rod, Pole and Wire hanging test at different time points before or after MPTP administration. The data are expressed as mean \pm SEM $(n=5)$. ${ }^{*} p<0.05,{ }^{* *} p<0.01,{ }^{* * *} p<0.001$ significant difference from control group, ${ }^{\sharp} p<0.05,{ }^{\#} p<0.01,{ }^{\# \#} p<0.001$ significant difference from MPTP treated group.

formance in the wire hanging test was significantly improved by $15 \mathrm{mg} / \mathrm{kg} \mathrm{AKNS}-2$ at $2 \mathrm{~h}$ and $24 \mathrm{~h}$ (as shown in Fig. 6D).

\section{Protective effects of AKNS-2 on mice damaged by MPTP administration}

DA is a neurotransmitter that transmits signals to other nerve cells in the brain. Dopaminergic neuron damage leads the loss of DA, thereby causing the motor symptoms of PD. The DA level in the ST was measured using an ELISA kit (Abnova). The result (as shown in Fig. 7A) indicated that the DA level in the ST was significantly reduced by $30 \mathrm{mg} / \mathrm{kg}$ MPTP administration. Interestingly, the MPTP-induced decrease in DA level was restored by ropinirole $(5 \mathrm{mg} / \mathrm{kg}$ ) and AKNS-2 (15 $\mathrm{mg} / \mathrm{kg}$ ). This result suggested that AKNS-2 protects dopaminergic neurons from the damage induced by MPTP.

MPTP can be converted to MPP ${ }^{+}$by MAO-B in glial cells, and $\mathrm{MPP}^{+}$is the actual toxin that damages dopaminergic neurons. MAO-B inhibitors prevent the metabolism of MPTP to $\mathrm{MPP}^{+}$by blocking the action of MAO-B. The present research tried to examine whether AKNS-2 protects dopaminergic neurons from the toxicity of MPTP by inhibiting MAO-B activity. MAO-B activities in the SN and ST were detected by a MAO-B assay kit (Promega). The result showed that MPTP (30 mg/ $\mathrm{kg}$ ) significantly enhanced the MAO-B activities in both the ST (Fig. 7B) and SN (Fig. 7C). However, AKNS-2 (5 and $15 \mathrm{mg} /$ $\mathrm{kg}$ ) failed to reduce the MPTP-enhanced MAO-B activities in both the ST and SN. This fact indicates that AKNS-2 is not an efficient MAO-B inhibitor and that the protective effect of AKNS-2 on MPTP-damaged mice is not due to MAO-B inhibition.

The MPTP-induced PD model is characterized by a decrease in $\mathrm{TH}$ in dopaminergic neurons. LBs are one of the pathologic features of $P D$, and $\alpha$-synuclein is the primary constituent of LBs. The present research measured the ex- pression of TH and $\alpha$-synuclein in the ST and SN of MPTPdamaged mice by Western blot analysis. Fig. 7D shows representative immunoblots of $\mathrm{TH}$ and $\alpha$-synuclein in the $\mathrm{ST}$ and $\mathrm{SN}$. TH levels were significantly reduced in the MPTP-treated group $(30 \mathrm{mg} / \mathrm{kg}$ ) compared with the normal control group in both the ST (Fig. 7E) and SN (Fig. 7F). Interestingly, the reduction in TH levels in the ST and SN was significantly reversed by the administration of the positive control ropinirole ( $5 \mathrm{mg} / \mathrm{kg}$ ) and 5 and $15 \mathrm{mg} / \mathrm{kg}$ AKNS-2. As shown in Fig. 7G and $7 \mathrm{H}, \alpha$-synuclein levels were significantly increased by MPTP administration in both the ST and SN, and a significant decrease in the $\alpha$-synuclein level was observed with the positive control ropinirole at $5 \mathrm{mg} / \mathrm{kg}$ compared with MPTP. Moreover, a marked reduction in $\alpha$-synuclein levels were also found in the ST and SN of AKNS-2-treated (5 and $15 \mathrm{mg} / \mathrm{kg}$ ) mice compared with MPTP-treated mice.

\section{Autophagy induction by AKNS-2 in an MPTP-induced in vivo PD model}

AKNS-2 induces autophagy in SH-SY5Y cells, and it protects cells from $\mathrm{MPP}^{+}$-induced cytotoxicity through autophagy activation. In our MPTP-induced in vivo PD model, we tried to examine the effect of AKNS-2 on autophagy regulation. Seven days after treatment with AKNS-2 and MPTP, autophagy-related protein markers in the ST and SN were measured by Western blot analysis. Fig. 8A and $8 \mathrm{~B}$ show representative immunoblots and the relative intensities of the protein markers in the ST and SN, respectively. The results indicated that MPTP enhanced LC3-II expression to a certain extent, but no significant difference was observed in the ST and ST. Compared with the MPTP treatment, the $15 \mathrm{mg} / \mathrm{kg}$ AKNS-2 treatment induced a significant increase in LC3-II expression in the ST. Enhanced LC3-II expression was found in the $15 \mathrm{mg} / \mathrm{kg}$ AKNS-2-treated group in the $\mathrm{SN}$, even though no significant difference was ob- 

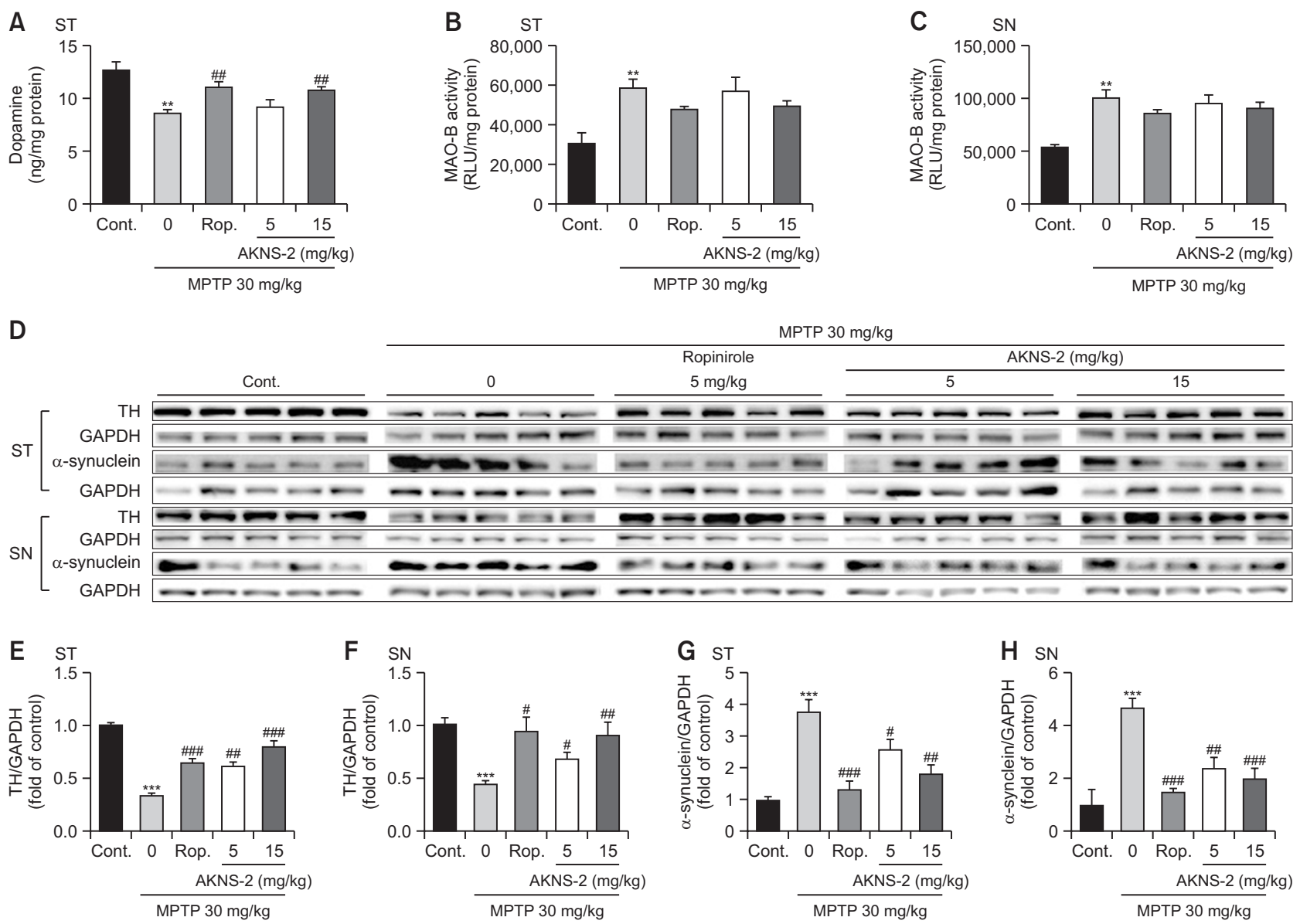

Fig. 7. Protective effect of AKNS-2 on MPTP-induced sub-chronic in vivo PD model. Seven days after MPTP injection, mice brain tissues of substantia nigra (SN) and striatum (ST) were dissected for biochemical analysis. (A) shows the dopamine level of ST from each group. (B) and (C) show MAO-B activities of ST and SN, respectively. (D) shows the typical protein bands of TH and $\alpha$-synuclein in ST and SN, respectively. (E) and (F) show the relative densities of TH from ST and SN, (G) and (H) show the relative densities of $\alpha$-synuclein from ST and $\mathrm{SN}$, respectively. The data are expressed as mean \pm SEM $(n=5) .{ }^{* *} p<0.01,{ }^{* * *} p<0.001$ significant difference from control group, ${ }^{*} p<0.05$, $\#^{\prime \prime} p<0.01, \ldots+0.001$ significant difference from MPTP treated group.

served. Compared with the normal group, the MPTP-treated group showed a significant decrease in p62 expression in the ST, and significantly reduced p62 levels were observed in both the ST and SN in the 5 and $15 \mathrm{mg} / \mathrm{kg}$ AKNS-2-treated groups compared with MPTP-treated group. Additionally, MPTP increased p-AMPK expression levels in both the ST and SN. Compared with the MPTP treatment, treatment with ropinirole and AKNS-2 led to significant increases in p-AMPK in both the ST and SN. Moreover, p-Erk expression in the ST and SN was decreased by $30 \mathrm{mg} / \mathrm{kg}$ MPTP treatment compared with the control condition and dramatically enhanced by $15 \mathrm{mg} / \mathrm{kg}$ AKNS-2 treatment compared with MPTP treatment. Similar to p-Erk expression, $p-U L K$ levels in the ST and SN were markedly reduced by MPTP administration, and the ropinirole and AKNS-2 (5 and $15 \mathrm{mg} / \mathrm{kg}$ ) treatments counteracted the effect of MPTP and significantly enhanced $p$-ULK expression in the ST and SN. We also measured p-mTOR expression in mice, and no significant difference was found in the ST or SN between the normal and MPTP-treated groups. Ropinirole and AKNS-2 obviously tended to reduce p-mTOR expression in the ST, even though no significant reduction was observed.
In the SN, significant reductions in p-mTOR were found in the ropinirole- and AKNS-2-treated groups.

\section{DISCUSSION}

$\mathrm{PD}$ is one of the most common neurodegenerative disorders, it is characterized by neuronal cell loss within the SN and formation of LBs due to deficient expression of genes and misfolded $\alpha$-synuclein (Davie, 2008). Autophagy was reported to efficiently degrade $\alpha$-synuclein, and the inhibition of autophagy induces the accumulation of insoluble $\alpha$-synuclein (Vogiatzi et al., 2008). Many current PD drugs have failed to prevent the degeneration of dopaminergic neurons (Mythri et al., 2012). Natural products, including herbal extracts, phytochemicals and bioactive ingredients, are believed to be potent sources of drugs for PD therapy (Mythri et al., 2012). The native Korean plant AKNS is a valuable herb to treat several diseases (Kim et al., 2018). The present study aimed to investigate the effect of a triterpene saponin, astersaponin I (named AKNS-2) derived from AKNS on autophagy activation and then to examine 
A

MPTP $30 \mathrm{mg} / \mathrm{kg}$
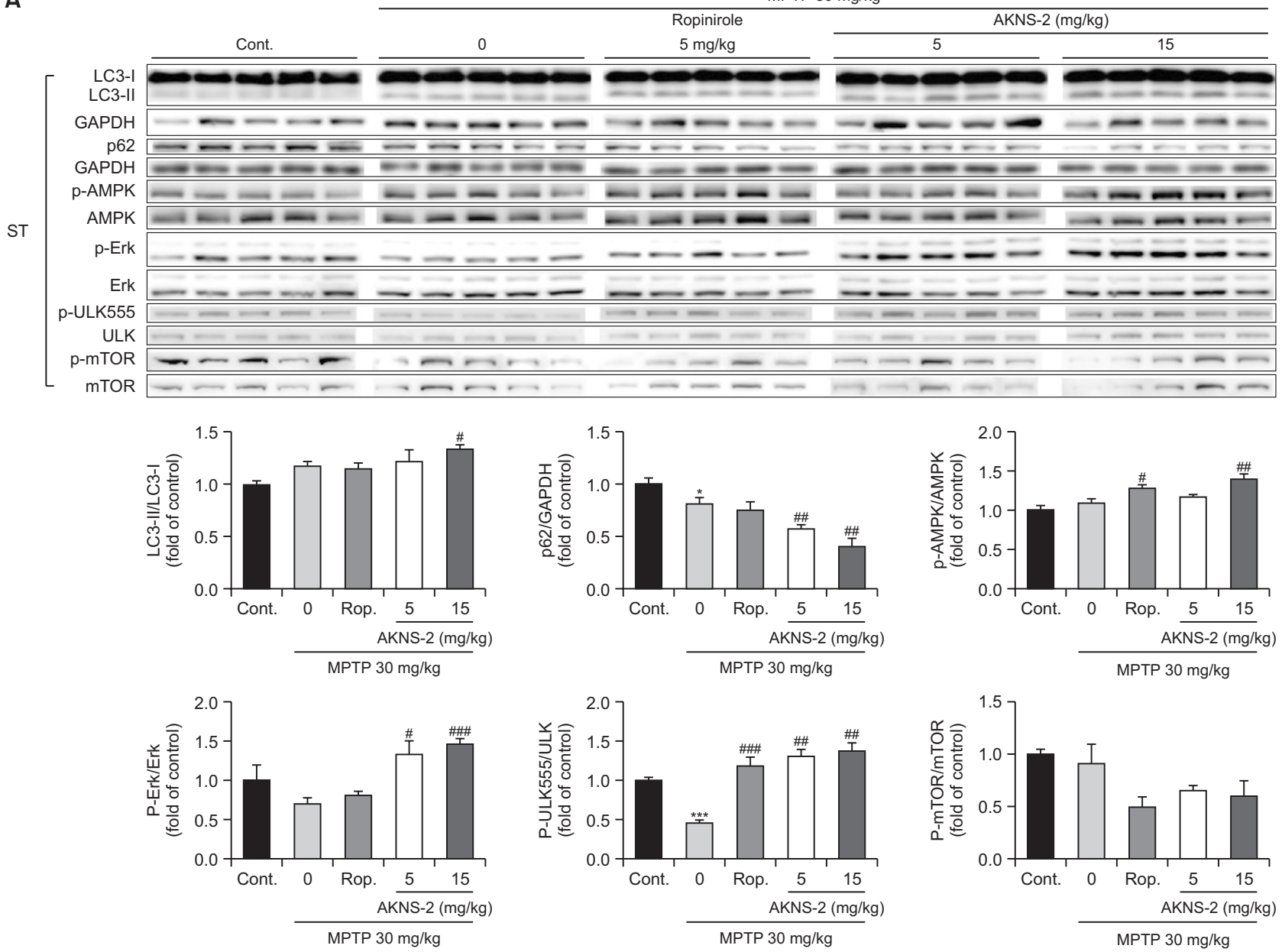

Fig. 8. Autophagy induction of AKNS-2 in in vivo experiment. (A) shows the typical protein bands and the relative densities of autophagy related protein markers including LC3, p62, AMPK, Erk, ULK and mTOR in striatum. (B) shows the typical protein bands and the relative densities of autophagy related protein markers including LC3, p62, AMPK, Erk, ULK and mTOR in substantia nigra. The data are expressed as mean \pm SEM $(n=5) .{ }^{*} p<0.05,{ }^{* *} p<0.01,{ }^{* *} p<0.001$ significant difference from control group, ${ }^{\#} p<0.05,{ }^{\#} p<0.01,{ }^{\# \#} p<0.001$ significant difference from MPTP treated group.

its beneficial effect on PD models. The autophagy enhancing effect of AKNS-2 were examined in in vitro experiments at various concentrations. The results showed that the AKNS2 significantly increased the expression of LC3-II, which reflects the amount of autophagosomes and autophagy-related structures (Yoshii and Mizushima, 2017). Next, cytotoxicity was measured by MTT assay in SH-SY5Y cells. AKNS-2 did not show significant cytotoxicity up to $40 \mu \mathrm{M}$. To confirm the autophagy-activating effect of AKNS-2, an autophagy inhibitor 3-MA, which inhibits the formation of autophagosomes by blocking class III PI3K, was applied to block autophagic flux (Ito et al., 2007). In addition to LC3-II, another important autophagy marker p62, which links LC3 and other ubiquitinated substrates was tested to verify autophagy activation (Zhang et al., 2013; Yoshii and Mizushima, 2017). The results revealed that AKNS-2 significantly enhanced LC3-II levels and inhibited p62 levels were dramatically reversed by 3-MA. Furthermore, we also carried out the mRFP-GFP-LC3 tandem fluorescent protein quenching assay to monitor autophagic flux (Zhang et al., 2013; Yoshii and Mizushima, 2017). Wortmanin (Wort 50
$\mathrm{nM}$ ) and Bafilomycin A1 (Baf $100 \mathrm{nM}$ ) were employed to inhibit the autophagic flux, respectively. AKNS-2-treatment dramatically increased cellular green and red puncta compared with control group, but the PI3K inhibitor Wort reduced green and red fluorescently labeled LC3 puncta. Moreover, Baf, an autophagy inhibitor which interferes green fluorescence quenching and autophagosome degradation (Zhang et al., 2013), produced more green and less red puncta than AKNS-2-treated group. These facts indicated AKNS-2-induced autophagic flux was inhibited, thus, AKNS-2 was confirmed as a potent autophagy inducer.

To explore the underlying pathways that AKNS-2 activates autophagy, we measured other autophagy-related protein markers except LC3-II. We found that AKNS-2 significantly enhance the cellular expression of p-AMPK, p-Erk, p-ULK555, but reduced p-mTOR expression. Supportably, phosphorylated AMPK, Erk upregulate autophagy via inhibiting mTOR, thus enhanced the phosphorylation of ULK (Inoki et al., 2003; Meley et al., 2006; Ganley et al., 2009; Jung et al., 2009; Yang et al., 2013; Hu et al., 2017b). Thus, AKNS-2 may activate au- 
B
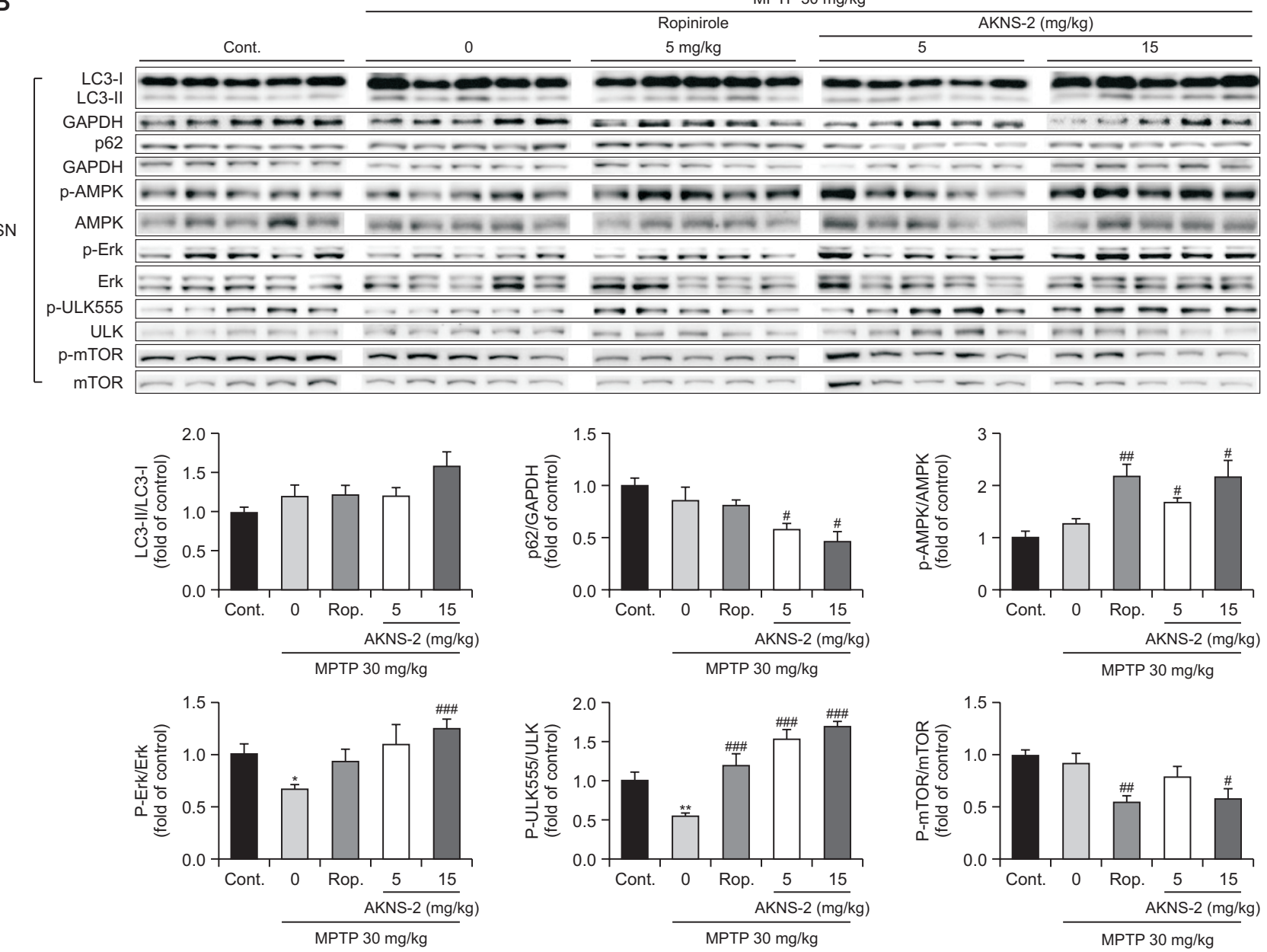

Fig. 8. Continued.

tophagy by modulating the Erk/mTOR and AMPK/mTOR pathways. To clarify the involved autophagy pathways induced by AKNS-2, the Erk inhibitor U0126 was used to block Erk signaling in SH-SY5Y cells. We found that AKNS-2 enhanced p-Erk expression was significantly inhibited by U0126. Moreover, the AKNS-2-induced changes in the expression of downstream autophagy regulators, including p-mTOR and p-ULK555, were reversed by $\mathrm{U} 0126$ which subsequently induced a decrease in the ratio of LC3-II/LC3-I. Therefore, AKNS-2 may upregulate autophagy at least partly by modulating the Erk/mTOR pathway. Additionally, the interference of AMPK signaling with siRNA abolished the enhancement of AKNS-2 on p-AMPK, thus reversed the expressions of downstream markers of $\mathrm{mTOR}$, p-ULK555 and LC3-II. These facts suggest that AKNS-2 may also activate autophagy by the AMPK/mTOR pathway.

It is well-known that MPTP, can be converted to the actual neurotoxin, $\mathrm{MPP}^{+}$, by MAO-B in astrocytes, $\mathrm{MPP}^{+}$blocks the mitochondrial respiratory chain and decreases cellular energy, thereby leading to an increase in oxidative stress and cell death (Ransom et al., 1987; Janhom and Dharmasaroja, 2015). Thus, MPP+-induced PD model in SH-SY5Y cells was used to test the neuro-protective effect of AKNS-2. TH, the rate-limiting enzyme in DA synthesis throughout the cen- tral nervous system (Tabrez et al., 2012; Liang et al., 2016), was examined as an index to monitor the protective effect of AKNS-2. The results suggested that TH level was significantly reduced by $\mathrm{MPP}^{+}$, but AKNS-2 counteracted the effect of $\mathrm{MPP}^{+}$and significantly restored the expression of $\mathrm{TH}$. Meanwhile. We found significantly increased LC3-II and deceased p62 expression by AKNS-2 treatment, which indicated the autophagy activation. Interestingly, the changed expressions of TH, LC3-II and p62 were reversed when autophagy was inhibited by 3-MA, which suggested that the protective effect of AKNS-2 was attributed to its autophagy-inducing activity. Furthermore, we tried to examine whether Erk/mTOR and AMPK/mTOR pathways involved in the protective effect of AKNS-2 on the MPP+-induced in vitro PD model. Western blot analysis showed that AKNS-2 and MPP ${ }^{+}$induced changes of LC3-II expression was significantly reversed by Erk inhibitor U0126, which suggested that AKNS-2-induced autophagy was blocked by U0126. Additionally, U0126 abolished the enhancement of cell viability by AKNS-2 in $\mathrm{MPP}^{+}$damaged SH-SY5Y cells. Similar results were found when we measured the levels of $\mathrm{TH}$ and $\alpha$-synuclein. AKNS-2 reversed the impaired expression of $\mathrm{TH}$ and $\alpha$-synuclein induced by MPP ${ }^{+}$, but U0126 abolished the action of AKNS-2. These results 
indicated that AKNS-2 protects against MPP+-induced neurotoxicity via Erk-activated autophagy. To investigate the involvement of AMPK signaling, AMPK signaling was disturbed by the transfection of AMPK siRNA in SH-SY5Y cells. Results showed AKNS-2 and $\mathrm{MPP}^{+}$induced expression change in LC3-II was significantly reversed by AMPK siRNA, which suggested that autophagy was blocked by AMPK siRNA. Meanwhile, AMPK disturbance abolished the AKNS-2 improved cell viability and $\mathrm{TH}, \alpha$-synuclein expressions in $\mathrm{MPP}^{+}$damaged SH-SY5Y cells, which suggested a protective effect of AKNS2 on the MPP ${ }^{+}$-induced in vitro PD model via AMPK-activated autophagy.

Since in vitro experiments demonstrated that AKNS-2 is a potent autophagy inducer and that autophagy activation protects neuronal cells from the toxicity of $\mathrm{MPP}^{+}$. Next, we tried to test the protective effect of AKNS-2 on an MPTP-induced PD mouse model. The loss of dopaminergic neurons leads to a decrease in DA in the ST, which results in motor disorders, including tremor, rigidity, bradykinesia and postural instability (Taylor et al., 2010). After habituation, C57BL/6j mice were subjected to a series of behavioral training regimen (rotarod test, pole test and wire hanging test). Mice behavioral performance were tested at the following three time points: 2 h, 24 $\mathrm{h}$, and $48 \mathrm{~h}$ after MPTP (30 mg/kg) injection. Performance in the rotarod test and the wire hanging test was dramatically impaired by MPTP injection, however, $15 \mathrm{mg} / \mathrm{kg}$ AKNS-2 significantly improved the impaired performance induced by MPTP in the rotarod and wire hanging tests at 2 and $24 \mathrm{~h}$ after MPTP injection. In the pole test, 5 and $15 \mathrm{mg} / \mathrm{kg}$ AKNS-2 only alleviated the MPTP-induced behavior impairment at $2 \mathrm{~h}$ after the MPTP injection. The behavioral tests in the present research were used to investigate the balance, muscle strength, and motor coordination of animals, and the results reflected the enhancing effect of AKNS-2 on MPTP-impaired mouse behavioral performance.

DA is an important neurotransmitter among of nerve cells in the brain, but it is not sufficiently produced when dopaminergic neurons loss was induced by MPTP, thereby leading to the decrease in DA synthesis and motor disorders found in PD patients (Poewe et al., 2017). We measured the DA level in the ST of mice 7 days after MPTP administration. The striatal DA level was significantly reduced by MPTP, and the decrease in DA was restored by $5 \mathrm{mg} / \mathrm{kg}$ positive control ropinirole and $15 \mathrm{mg} / \mathrm{kg}$ AKNS-2. The enhancing effect of AKNS-2 on the DA level explained the reason why AKNS-2 improved the mouse behavioral performance damaged by MPTP. Moreover, MPTP crosses the BBB and is converted into $\mathrm{MPP}^{+}$, the actual neurotoxin, by MAO-B in astrocytes (Kupsch et al., 2001; Mallajosyula et al., 2008). Thus, inhibiting MAO-B activity may also alleviate the toxicity of MPTP and prevent the loss of dopaminergic neurons. The present study tried to clarify whether the protection of AKNS-2 on the MPTP-induced PD model relates to MAO-B activity. The MAO-B activities in the ST and $\mathrm{SN}$ were examined by a MAO-B assay kit (Promega), the results did not show any significant difference in MAO-B activity between the MPTP- and AKNS-2-treated groups, which indicated that AKNS-2 was not an efficient MAO-B inhibitor.

We also measured the TH and $\alpha$-synuclein levels in the ST and SN from mice treated with MPTP and AKNS-2. Consistent with the results of the in vitro experiment, MPTP markedly reduced TH levels and enhanced $\alpha$-synuclein accumulation in both the ST and SN. However, the changes in the expression of TH and $\alpha$-synuclein induced by MPTP were significantly reversed by AKNS-2, which indicated the protective effect of AKNS-2 against MPTP-induced toxicity. Additionally, we investigated autophagy activation by measuring the expression of LC3-II and p62 in mouse brain using Western blot analysis. The results showed that LC3-II expression in the ST was significantly enhanced by AKNS-2, while the LC3-II level obviously tended to increase in SN. Meanwhile, AKNS-2 significantly decreased p62 levels in ST and SN. Based on these data, autophagy was activated in the in vivo PD model. Subsequently, other protein markers related to autophagy regulation were examined, and results were consistent with the in vitro experiment, which showed that AKNS-2 significantly upregulated p-AMPK, p-Erk, and p-ULK555 levels and inhibited pmTOR levels. The upregulated autophagy induced by AKNS-2 is probably due to the activation of the AMPK/mTOR and Erk/ mTOR pathways and exerts a protective effect on the MPTPinduced in vivo PD model. Autophagy-lysosomal pathway is the one of the major ways for protein degradation in neuron, and it is responsible for the degradation of abnormal aggregated proteins including $\alpha$-synuclein (Le, 2020). In the present research, AKNS-2 activates the Erk/mTOR and AMPK/mTOR signaling and enhances the transfer of $\alpha$-synuclein to lysosome by forming the autophagosome, eventually promotes the degradation of $\alpha$-synuclein and improves the symptoms found in MPTP induced PD models.

The present study reported a novel active compound, AKNS-2, was isolated from natural plant Aster koraiensis. We systematically studied the autophagy inducing effect and neuro-protective effect of AKNS-2. Data from the in vitro experiments demonstrated that AKNS-2 exerts a prominent autophagy-inducing effect by activating Erk and AMPK signaling, meanwhile, AKNS-2 showed excellent neuro-protective effects on $\mathrm{MPP}^{+}$induced in vitro and MPTP induced in vivo PD model through autophagy activation, it may serve as a candidate for PD therapy.

\section{CONFLICT OF INTEREST}

The authors declared no conflict of interest exists.

\section{ACKNOWLEDGMENTS}

This work was funded and supported by the Bio-Synergy Research Project (NRF-2012M3A9C4048793) and the Bio \& Medical Technology Development Program (NRF2015M3A9A5030735) of the Ministry of Science, ICT, and Future Planning through the National Research Foundation of the Republic of Korea to HOY. This work also was supported by Korea Institute of Science and Technology (KIST) Institutional Program (2Z05640).

\section{REFERENCES}

Bjørkøy, G., Lamark, T., Pankiv, S., Øvervatn, A., Brech, A. and Johansenet, T. (2009) Monitoring autophagic degradation of p62/ SQSTM1. Methods Enzymol. 452, 181-197.

Choi, D. Y., Lee, M. K. and Hong, J. T. (2013) Lack of CCR5 modifies glial phenotypes and population of the nigral dopaminergic 
neurons, but not MPTP-induced dopaminergic neurodegeneration. Neurobiol. Dis. 49, 159-168.

Chu, Y., Dodiya, H., Aebischer, P., Olanow, C. W. and Kordower, J. H. (2009) Alterations in lysosomal and proteasomal markers in Parkinson's disease: relationship to alpha-synuclein inclusions. $\mathrm{Neu}-$ robiol. Dis. 35, 385-398.

Conway, K. A., Lee, S. J., Rochet, J. C., Ding, T. T., Harper, J. D. Williamson, R. E. and Lansbury, P. T., Jr. (2000) Accelerated oligomerization by Parkinson's disease linked alpha-synuclein mutants. Ann. N. Y. Acad. Sci. 920, 42-45.

Dauer, W. and Przedborski, S. (2003) Parkinson's disease: mechanisms and models. Neuron 39, 889-909.

Davie, C. A. (2008) A review of Parkinson's disease. Br. Med. Bull. 86, $109-127$.

Dehay, B., Bove, J., Rodriguez-Muela, N., Perier, C., Recasens, A., Boya, P. and Vila, M. (2010) Pathogenic lysosomal depletion in Parkinson's disease. J. Neurosci. 30, 12535-12544.

Egan, D. F., Shackelford, D. B., Mihaylova, M. M., Gelino, S. R., Kohnz, R. A., Mair, W., Vasquez, D. S., Joshi, A., Gwinn, D. M., Taylor, R., Asara, J. M., Fitzpatrick, J., Dillin, A., Viollet, B., Kundu, M., Hansen, M. and Shaw, R. J. (2011) Phosphorylation of ULK1 (hATG1) by AMP-activated protein kinase connects energy sensing to mitophagy. Science 331, 456-461.

Ganley, I. G., Lam du, H., Wang, J., Ding, X., Chen, S. and Jiang, X (2009) ULK1.ATG13.FIP200 complex mediates mTOR signaling and is essential for autophagy. J. Biol. Chem. 284, 12297-12305.

Ghosh, R., Gilda, J. E. and Gomes, A. V. (2014) The necessity of and strategies for improving confidence in the accuracy of western blots. Expert Rev. Proteomics 11, 549-560.

Glick, D., Barth, S. and Macleod, K. F. (2010) Autophagy: cellular and molecular mechanisms. J. Pathol. 221, 3-12.

$\mathrm{He}, \mathrm{C}$. and Klionsky, D. J. (2009) Regulation mechanisms and signaling pathways of autophagy. Annu. Rev. Genet. 43, 67-93.

Hu, X., Song, Q., Li, X., Li, D. D., Zhang, Q., Meng, W. H. and Zhao, Q. C. (2017a) Neuroprotective effects of Kukoamine A on neurotoxin-induced Parkinson's model through apoptosis inhibition and autophagy enhancement. Neuropharmacology 117, 352-363.

Hu, X. X., Shi, S., Wang, H., Yu, X., Wang, Q., Jiang, S., Ju, D., Ye, L. and Feng, M. (2017b) Blocking autophagy improves the anti-tumor activity of afatinib in lung adenocarcinoma with activating EGFR mutations in vitro and in vivo. Sci. Rep. 7, 4559.

Hyun, S.-W., Kim, J., Jo, K., Kim, J. S. and Kim, C. S. (2018) Aster koraiensis extract improves impaired skin wound healing during hyperglycemia. Integr. Med. Res. 7, 351-357.

Inoki, K., Zhu, T. and Guan, K. L. (2003) TSC2 mediates cellular energy response to control cell growth and survival. Cell 115, 577-590.

Ito, S., Koshikawa, N., Mochizuki, S. and Takenaga, K. (2007) 3-Methyladenine suppresses cell migration and invasion of HT1080 fibrosarcoma cells through inhibiting phosphoinositide 3-kinases independently of autophagy inhibition. Int. J. Oncol. 31, 261-268.

Janhom, P. and Dharmasaroja, P. (2015) Neuroprotective effects of alpha-mangostin on MPP(+)-induced apoptotic cell death in neuroblastoma SH-SY5Y cells. J. Toxicol. 2015, 919058.

Jung, C. H., Jun, C. B., Ro, S. H., Kim, Y. M., Otto, N. M., Cao, J., Kundu, M. and Kim, D. H. (2009) ULK-Atg13-FIP200 complexes mediate mTOR signaling to the autophagy machinery. Mol. Biol. Cell 20, 1992-2003.

Kim, J., Kundu, M., Viollet, B. and Guan, K. L. (2011) AMPK and mTOR regulate autophagy through direct phosphorylation of Ulk1. Nat. Cell Biol. 13, 132-141.

Kim, J., Lee, Y. M., Jung, W., Park, S. B., Kim, C. S. and Kim, J. S. (2018) Aster koraiensis extract and chlorogenic acid inhibit retinal angiogenesis in a mouse model of oxygen-induced retinopathy. Evid. Based Complement. Alternat. Med. 2018, 6402650.

Klaidman, L.K., Adams, J. D., Jr., Leung, A. C., Kim, S. S. and Cadenas, E. (1993) Redox cycling of MPP+: evidence for a new mechanism involving hydride transfer with xanthine oxidase, aldehyde dehydrogenase, and lipoamide dehydrogenase. Free Radic. Biol. Med. 15, 169-179.

Kostrzewa, R. M. (2014) Handbook of Neurotoxicity. Springer, New York.

Kupsch, A., Sautter, J., Götz, M. E., Breithaupt, W., Schwarz, J., Youdim,
M. B., Riederer, P., Gerlach, M. and Oertel, W. H. (2001) Monoamine oxidase-inhibition and MPTP-induced neurotoxicity in the non-human primate: comparison of rasagiline (TVP 1012) with selegiline. J. Neural Transm. (Vienna) 108, 985-1009.

Kwon, J., Ko, K., Zhang, L., Zhao, D., Yang, H. O. and Kwon, H. C. (2019) An autophagy inducing triterpene saponin derived from Aster koraiensis. Molecules 24, 4489.

Liang, J. Q., Wang, L., He, J. C. and Hua, X. (2016) Verbascoside promotes the regeneration of tyrosine hydroxylase-immunoreactive neurons in the substantia nigra. Neural Regen. Res. 11, 101-106.

Le, W. (2020) Autophagy: Biologyand Disease- Clinical Science. Springer, Singapore.

Mallajosyula, J. K., Kaur, D., Chinta, S. J., Rajagopalan, S., Rane, A., Nicholls, D. G., Di Monte, D. A., Macarthur, H. and Andersen, J. K. (2008) MAO-B elevation in mouse brain astrocytes results in Parkinson's pathology. PLOS ONE 3, e1616.

Meley, D., Bauvy, C., Houben-Weerts, J. H. P. M., Dubbelhuis, P. F., Helmond, M. T. J., Codogno, P. and Meijer, A. J. (2006) AMP-activated protein kinase and the regulation of autophagic proteolysis. J. Biol. Chem. 281, 34870-34879.

Moscat, J. and Diaz-Meco, M. T. (2009) p62 at the crossroads of autophagy, apoptosis, and cancer. Cell 137, 1001-1004.

Mythri, R. B., Harish, G. and Bharath, M. M. (2012) Therapeutic potential of natural products in Parkinson's disease. Recent Pat. Endocr. Metab. Immune Drug Discov. 6, 181-200.

Nashatizadeh, M. M., Lyons, K. E. and Pahwa, R. (2009) A review of ropinirole prolonged release in Parkinson's disease. Clin. Interv. Aging 4, 179-186.

Noda, T. and Ohsumi, Y. (1998) Tor, a phosphatidylinositol kinase homologue, controls autophagy in yeast. J. Biol. Chem. 273, 39633966.

Pattingre, S., Bauvy, C. and Codogno, P. (2003) Amino acids interfere with the ERK1/2-dependent control of macroautophagy by controlling the activation of Raf-1 in human colon cancer HT-29 cells. J. Biol. Chem. 278, 16667-16674.

Poewe, W., Seppi, K., Tanner, C. M., Halliday, G. M., Brundin, P., Volkmann, J., Schrag, A. E. and Lang, A. E. (2017) Parkinson disease. Nat. Rev. Dis. Primers 3, 17013.

Prabakaran, M., Kim, S. H., Mugila, N., Hemapriya, V., Parameswari, K., Chitra, S. and Chunga, I. M. (2017) Aster koraiensis as nontoxic corrosion inhibitor for mild steel in sulfuric acid. J. Ind. Eng. Chem. 52, 235-242.

Ramsay, R. R. and Singer, T. P. (1986) Energy-dependent uptake of Nmethyl-4-phenylpyridinium, the neurotoxic metabolite of 1-methyl4-phenyl-1,2,3,6-tetrahydropyridine, by mitochondria. J. Biol. Chem. 261, 7585-7587.

Ransom, B. R., Kunis, D. M., Irwin, I. and Langston, J. W. (1987) Astrocytes convert the parkinsonism inducing neurotoxin, MPTP, to its active metabolite, MPP ${ }^{+}$. Neurosci. Lett. 75, 323-328.

Sarkar, S. (2013) Regulation of autophagy by mTOR-dependent and mTOR-independent pathways: autophagy dysfunction in neurodegenerative diseases and therapeutic application of autophagy enhancers. Biochem. Soc. Trans. 41, 1103-1130.

Tabrez, S., Jabir, N. R., Shakil, S., Greig, N. H., Alam, Q., Abuzenadah, A. M., Damanhouri, G. A. and Kamal, M. A. (2012) A synopsis on the role of tyrosine hydroxylase in Parkinson's disease. CNS Neurol. Disord. Drug Targets 11, 395-409.

Taylor, T. N., Greene, J. G. and Miller, G. W. (2010) Behavioral phenotyping of mouse models of Parkinson's disease. Behav. Brain Res. 211, 1-10.

Vogiatzi, T., Xilouri, M., Vekrellis, K. and Stefanis, L. (2008) Wild type alpha-synuclein is degraded by chaperone-mediated autophagy and macroautophagy in neuronal cells. J. Biol. Chem. 283, 2354223556.

Wang, J., Whiteman, M. W., Lian, H., Wang, G., Singh, A., Huang, D. and Denmark, T. (2009) A non-canonical MEK/ERK signaling pathway regulates autophagy via regulating Beclin 1. J. Biol. Chem. 284, 21412-21424.

Wu, Y. T., Tan, H. L., Shui, G., Bauvy, C., Huang, Q., Wenk, M. R., Ong, C. N., Codogno, P. and Shen, H. M. (2010) Dual role of 3-methyladenine in modulation of autophagy via different temporal patterns of inhibition on class I and III phosphoinositide 3-kinase. J. Biol. 
Chem. 285, 10850-10861.

Yang, Y. H., Chen, K., Li, B., Chen, J. W., Zheng, X. F., Wang, Y. R., Jiang, S. D. and Jiang, L. S. (2013) Estradiol inhibits osteoblast apoptosis via promotion of autophagy through the ER-ERK-mTOR pathway. Apoptosis 18, 1363-1375.

Yoshii, S. R. and Mizushima, N. (2017) Monitoring and measuring autophagy. Int. J. Mol. Sci. 18, 1865.
Zhang, X. J., Chen, S., Huang, K. X. and Le, W. (2013) Why should autophagic flux be assessed? Acta Pharmacol. Sin. 34, 595-599.

Zhu, W., Gao, Y., Wan, J., Lan, X., Han, X., Zhu, S., Zang, W., Chen, X., Ziai, W., Hanley, D. F., Russo, S. J., Jorge, R. E. and Wang, J. (2018) Changes in motor function, cognition, and emotion-related behavior after right hemispheric intracerebral hemorrhage in various brain regions of mouse. Brain Behav. Immun. 69, 568-581. 